\title{
The Spectral Gap for Some Spin Chains with Discrete Symmetry Breaking
}

\author{
Bruno Nachtergaele \\ Department of Physics, Princeton University, Princeton, NJ 08544-0708, USA. \\ E-mail: bxn@math.princeton.edu
}

Received: 2 November 1994/in revised form: 9 March 1995

\begin{abstract}
We prove that for any finite set of generalized valence bond solid (GVBS) states of a quantum spin chain there exists a translation invariant finite-range Hamiltonian for which this set is the set of ground states. This result implies that there are GVBS models with arbitrary broken discrete symmetries that are described as combinations of lattice translations, lattice reflections, and local unitary or anti-unitary transformations. We also show that all GVBS models that satisfy some natural conditions have a spectral gap. The existence of a spectral gap is obtained by applying a simple and quite general strategy for proving lower bounds on the spectral gap of the generator of a classical or quantum spin dynamics. This general scheme is interesting in its own right and therefore, although the basic idea is not new, we present it in a system-independent setting. The results are illustrated with a number of examples.
\end{abstract}

\section{Table of Contents}

1. Introduction and Statement of the Main Results .................. 566

2. Lower Bounds for the Spectral Gap - A General Strategy ............ 571

3. Some Basic Facts on Pure GVBS States ......................... 577

4. The Intersection Property of GVBS States $\ldots \ldots \ldots \ldots \ldots \ldots \ldots \ldots \ldots \ldots$

5. Existence of GVBS Interactions: the Proof of Theorem $1 \ldots \ldots \ldots \ldots \ldots .588$

6. Existence of the Spectral Gap: the Proof of Theorem 2 ............. 590

7. Examples, Counterexamples, and Open Problems .................. 594

Acknowledgements ..................................... 603

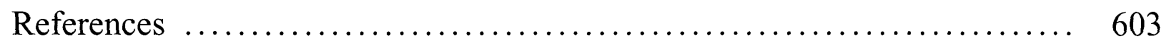

Copyright (c) 1994 by the author. Faithful reproduction of this article by any means is permitted for non-commercial purposes. 


\section{Introduction and Statement of the Main Results}

Due to recent progress made by various authors it has become clear that the variety of behaviour found in the ground states of quantum spin models is much larger than was expected before, even in one dimension. In particular there has been revived interest in models with a discrete symmetry breaking [1-6]. A good strategy for exploring this variety of phenomena has been, and still is, the study of simple exactly solvable models in as great detail as is possible. Therefore, various authors tried to construct models with explicitly known ground states that exhibit some interesting properties as, e.g., a specific kind of discrete symmetry breaking. E.g., in [7] Affleck, Arovas, Marston, and Rabson construct spin chains with nearest neighbour interactions that have ground states with broken charge conjugation symmetry. These ground states are given by means of a Generalized (or eXtended) Valence Bond Solid construction (GVBS, or XVBS, states).

The first question addressed in this paper is the following. Given a finite group of symmetry transformations of a quantum spin chain and a local observable (or a finite set of local observables) that distinguishes ground states with broken symmetry, can one always find a model with finite range interactions which has the prescribed symmetries and symmetry breaking ground states? The answer is positive: a model of the GVBS type with the desired properties can always be constructed (see Theorem 1 and the remarks following it at the end of this introduction).

It is widely believed that if a one-dimensional quantum spin model has a finite number of ground states (typically related to one another by a discrete symmetry) that all have a finite correlation length (i.e. exponential decay of correlations), then there is a spectral gap above the ground state energy that does not vanish in the thermodynamic limit. In some cases this is rather well understood in terms of the two-dimensional quantum field theory that describes the long-distance and low-energy behaviour of the spin chain [8]. The Lorentz invariance of the quantum field theory relates the correlation length $\xi$ in space with the gap $\Delta$ in the spectrum which governs the decay of correlations in (imaginary) time. The only intervening parameter is the spin-wave velocity $v$ which plays the role of the speed of light in the relativistic theory $[9,10]$ :

$$
\Delta=v / \xi .
$$

This argument is rather heuristic at this point and cannot be given the status of a mathematical proof. In fact, one should not expect that a unique or a finite number of ground states with a finite correlation length is always accompanied by a spectral gap. Certain exceptions to this rule of thumb occur, as we show in an example in Sect. 7.

To give a proof of the existence of a spectral gap in the GVBS models with discrete symmetry breaking is the second aim of this work. Theorem 2 states that under some simple conditions any quantum spin Hamiltonian with finite range interactions that has only a finite number of GVBS states as its ground states, indeed possesses a spectral gap. We also show in a counterexample (Example 2 in Sect. 7) that the conditions of Theorem 2 are necessary or at least that they cannot be completely omitted; there are quantum spin chains with nearest neighbour interactions and a finite number of GVBS ground states, that do not have a spectral gap in the thermodynamic limit.

The first proof (in an isotropic model) of the existence of a spectral gap was given by Affleck, Kennedy, Lieb, and Tasaki in $[11,12]$ in a model with 
a unique ground state (the AKLT model), and in a model with two groundstates (the Majumdar-Ghosh model). A different proof, which applies to all GVBS models with a unique ground state, was given by Fannes, Nachtergaele, and Werner in $[13,14]$. Apart from being more general, this proof has the advantage of providing reasonable lower bounds on the magnitude of the spectral gap. Good upper bounds, both for the AKLT chain and for the spin-1 Heisenberg antiferromagnetic chain, are usually quite easy to obtain due to the variational principle. For the AKLT chain such upper bounds were obtained in [15] and [16]. For quite some time already, there is also ample numerical evidence for the Haldane gap (see [17-23]. Knabe [16] also provides a general argument that, in combination with sufficiently good numerical estimates on the gap for finite volumes, also proves the existence of a gap in the thermodynamic limit. By now, very precise numerical estimates of the spectral gap of the AKLT chain are available $[24,10]$ due to the numerical algorithm developed by White $[25,26]$. Recently Kennedy obtained upper bounds of comparable precision [27] by a much simpler, variational method. Exact excited states in GVBS models are constructed only in special cases [28, 29].

The result (Theorem 2) of this paper is very much in the spirit of [14] in that it also provides, in principle, a reasonable estimate for the gap. It is more general because it covers the case of multiple (a finite number of) ground states. Part of the argument (Theorem 3), however, is different from [14] and is an elaboration of a discussion with H.-T. Yau. In Theorem 3 we present a general strategy for obtaining lower bounds on the spectral gap, which, we believe, could be of wider applicability. On a more formal level the basic structure of this argument seems to be present in all of the proofs of the existence of a spectral gap known to me, both for quantum spin Hamiltonians and for the generators of stochastic time evolutions of classical spin systems [30-33]. The work of Martinelli and Olivieri is reviewed in [37]. In the case of stochastic dynamics of classical spin systems the logarithmic Sobolev inequality plays an important role. It implies the exponential decay of correlations (and provides an estimate on the gap) [34]. Proofs of the logarithmic Sobolev inequality under a Dobrushin-Shlosman mixing condition and related properties for classical spin systems are given in [38-41,32]. The connection with the spectral gap is discussed in [36]. We do not know of a useful analogue of the logarithmic Sobolev inequality for quantum spin systems. There is, however, a natural mixing condition ( $\mathrm{C} 3$ and $\mathrm{C}^{\prime}$ in Sect. 2) that enters in our proof of the existence of a gap.

Theorem 3 is formulated in a system-independent setting which does not explicitly refer to the one-dimensionality of the system. It also brings the proofs of [12] and [14] closer together, retaining the best of both and at the same time making them more transparent. The same theorem can also be used to give lower bounds on the finite-volume gap in cases where the gap vanishes in the thermodynamic limit. One then obtains a lower bound on the rate at which the gap vanishes. In some cases the criterion for a non-vanishing spectral gap in the thermodynamic limit that is contained in Theorem 3 can be shown to be sharp. We refer the reader to Sect. 2 for a discussion of this general scheme and some related work.

The existence of a spectral gap in the generator of a classical or quantum spin dynamics, is an important property with direct relevance for the physical behaviour of the system. This is true for more general models than just one-dimensional quantum spin systems. It is, e.g., a fundamental ingredient in all theories of the fractional quantum Hall effect. (See e.g. the pseudopotential model of Haldane [42], and the 
work of Fröhlich and coworkers [43].) From the mathematical point of view there are very few techniques available to prove the existence of a gap. Also for this reason we chose to present the general strategy, employed in this work, in the form of an independent theorem (Sect. 2), hoping that inspiration for a proof of the spectral gap in other systems might be drawn from it.

In this paper we will be mainly dealing with a generalization of the so-called Valence-Bond-Solid models [44] that we will call GVBS models. GVBS models are special and one cannot expect them to reveal all properties that might be found in more general models. However there are quite a number of aspects in which they do provide new insight.

GVBS models are special, first of all, because their exact ground states can be constructed in an explicit way. In general this is not possible for quantum spin models, not even in one dimension. Moreover they have a very simple structure which is quite easy to picture and essentially involves only finite-dimensional objects. This is closely related with another special property that the GVBS models share: the energy is minimized locally in their ground states. This means that, from a certain finite length on, the minimum energy per bond in a finite interval is the same as the minimum energy per bond for the infinite system. On the level of the states this property is reflected in the fact that the finite volume ground states coincide with the restrictions to that finite volume of the infinite volume ground states. One can argue that for a generic (non-GVBS) interaction, the energy is not minimized locally. This is due to the non-triviality of the state extension problem for quantum spin chains [45].

Let us now look at what makes the GVBS models and their ground states interesting objects to study. As mentioned above, the first rigorous proof of the properties of the Haldane phase [46, 47] (in particular the existence of a spectral gap) was given by Affleck, Kennedy, Lieb and Tasaki in a particular spin-1 VBS chain $[12,11]$, which is by now called the AKLT-model, and it is fair to say that it served as a paradigm for many of the subsequent studies on massive quantum spin chains. In that paper the authors also gave a detailed analysis of some other VBS models [48-54], which had been studied in the literature before, and thus introduced a new class of quantum spin Hamiltonians for which exact ground states with nontrivial properties can be constructed. Since then various other VBS models were introduced [15, 44, 55-57], including some interesting two-dimensional models [5864]. A rather detailed analysis of correlation functions in a class of GVBS chains, including inhomogeneous ones, see $[65,66]$. In [14] the authors give a definition of Generalized Valence Bond Solid states (starting from a proposal for the construction of Quantum Markov Chains by Accardi [67, 68] and their analysis leads to a wide variety of VBS-type models. In particular the Generalized VBS models and the construction of their exact ground states does not rely on invariance under SU(2) or $\mathrm{SU}(\mathrm{N})$ or any other symmetry group, as was the case in all previous constructions. We will review this construction in a simplified form below. In [69] it is shown that this construction generates a weakly dense subset of the set of ergodic states. The paper [14] is strongly concentrated on models with a unique ground state. Here our aim is to extend the basic construction of GVBS Hamiltonians to the case where the ground state degeneracy is arbitrary but finite, and to give a proof for the existence of a spectral gap in that case. This is probably the most general situation where there is indeed a gap in one-dimensional GVBS models. One can show that the degeneracy of the ground states for a GVBS model (as defined in [14]) is either 
finite or grows exponentially fast with the volume, see [13]). We exclude this case from our discussion here.

In this paper by a Generalized VBS-model (GVBS-model) we mean a onedimensional model with a translation invariant or periodic interaction for which there exists a non-empty finite set of ground states that minimize the energy locally on some finite length scale and that all the states in this set can be obtained by the generalized VBS-construction given in $[14,70]$, where they are called purely generated $C^{*}$-finitely correlated states. Here, we prefer to start from scratch and introduce them in a way that is as close as possible to the traditional VBS-construction as it is known in the literature (see e.g. [11]). In fact this alternative description was already given in [14]. There exist models that satisfy our working definition of GVBS-model - for which the main justification is that it defines the class of models for which our theorems apply - in all respects except for the fact that the number of their zero-energy ground states is infinite and in particular contains non-translation invariant states. The general results presented in this paper do not apply to such models. In some cases they are not expected to have a gap [55], in other cases one can still show that there is a non-vanishing gap [71, 72].

Before stating the two main theorems of this paper we now review the GVBSconstruction and introduce the necessary definitions and notations.

We label the sites in the chain by integers $i \in \mathbb{Z}$ and with each site we associate a copy of the finite-dimensional Hilbert space $\mathbb{C}^{d}$, which we denote by $\mathscr{H}_{i}$ whenever its location in the chain is relevant. So, if one is to consider a chain of spin $s$ variables one has to take $d=2 s+1$. For any finite set $\Lambda \subset \mathbb{Z}$, define $\mathscr{H}_{\Lambda}=\otimes_{i \in \Lambda} \mathscr{H}_{i}$. Let $k \geqq 1$ be an integer and $W$ a linear map: $\mathbb{C}^{d} \rightarrow \mathbb{C}^{k} \otimes \mathbb{C}^{k}$, and let $\varphi$ be a unit vector in $\mathbb{C}^{k} \otimes \mathbb{C}^{k}$. For any finite interval $[M, N] \subset \mathbb{Z}$ we can define a state for that finite piece of the spin chain by giving its expectation values for all observables of the form $A=A_{1} \otimes \cdots \otimes A_{n}$, where for all $i, M \leqq i \leqq N, A_{i}$ is a $d \times d$ matrix with complex entries (e.g. a spin matrix located at the site $i$ ). In this paper, states (expectation values) are usually denoted by $\omega$ and scalar products by $\langle\cdot \mid \cdot\rangle$. The expectation value of $A$ is now defined by:

$\omega_{[M, N], \alpha, \beta}(A)=\frac{\left\langle\varphi^{\otimes N-M+2} \mid\left(e_{\alpha} \otimes W A_{M} W^{*} \otimes \cdots \otimes W A_{N} W^{*} \otimes e_{\beta}\right) \varphi^{\otimes N-M+2}\right\rangle}{\mathcal{N}}$,

where $\mathcal{N}$ is the normalization factor and $e_{\alpha}$ and $e_{\beta}$ are two non-negative definite $k \times k$ matrices that play the role of boundary conditions. It is implicitly assumed that the expression is not identically vanishing (e.g. $W$ should be different from zero). It can be shown that for any choice of $e_{\alpha}$ and $e_{\beta}$ there exists an integer $p \geqq 1$ such that for all $A$ the limit

$$
\omega_{\alpha, \beta}(A)=\lim _{M \rightarrow-\infty, N \rightarrow+\infty} \omega_{[p M, p N], \alpha, \beta}(A)
$$

exists and results in a well-defined state of the infinite chain. Typically, the state $\omega_{\alpha, \beta}$ is then $p$-periodic, i.e. invariant under translations in the chain over distances that are multiples of $p$. We will see in Sect. 3 how to determine the possible values of $p$ and also how the limit points can be described in a simple way. Sometimes it is convenient to regroup the chain, i.e. to partition the chain into intervals of length $p$, and to consider it as a new chain where the elementary sites are now groups of $p$ consecutive sites in the original chain. This is also a method to construct $p$ periodic states. All states of the chain obtained by the construction (1.1), possibly 
after carrying out a regrouping of the chain first, will be called Generalized Valence Bond Solid states (GVBS states). As an example one can think of the AKLT model. There $d=3$ and $k=2$, and $W$ identifies the space $\mathbb{C}^{3}$ with the subspace of $\mathbb{C}^{2} \otimes \mathbb{C}^{2}$ (where the two $\mathbb{C}^{2}$ are carrying a spin $\frac{1}{2}$ ), which corresponds to total spin $=1$.

By an interaction of range $l$ for a quantum spin chain we simply mean a selfadjoint element $h \in\left(\mathscr{M}_{d}\right)^{\otimes l}$. In this paper we will always assume that $h$ is nonnegative definite, which, by itself, is no restriction because additive constants only change the value of the ground state energy but not the ground states of the model. The Hamiltonian for a finite piece of the chain, say the interval $[M, N]$, is given by:

$$
H_{[M, N]}=\sum_{l=M}^{N-l+1} h_{l},
$$

where $h_{l}$ is a copy of $h$ located at the sites $i, i+1, \ldots, i+l-1$ of the chain. Here we are interested in situations where there exists at least one state $\omega$ of the infinite chain such that $\omega\left(h_{l}\right)=0$ for all $i \in \mathbb{Z}$, which, as was mentioned before, really is a rather special property. For a further discussion of models where this property is satisfied and a general theory about the set of ground states of such models as well as some non-GVBS examples, see [73]. For a given $h$, denote the set of all zero energy states of the chain by $\mathscr{F}_{h}$, and let us call this set the set of ground states of the model. It is obvious that $\mathscr{F}_{h}$ is a face: if three states of the chain $\omega, \eta_{1}$ and $\eta_{2}$, satisfy the relation $\omega=t \eta_{1}+(1-t) \eta_{2}$ for some $t \in(0,1)$, then $\omega \in \mathscr{F}_{h} \Leftrightarrow \eta_{1}, \eta_{2} \in \mathscr{F}_{h}$. The different ground states of the model (in the conventional sense) are the extreme points of $\mathscr{F}_{h}$, and they are pure states.

It can be shown that any GVBS state as defined above is a convex combination of a finite number of pure states that are necessarily also GVBS states: i.e. for any GVBS state $\omega$ there exist a finite number of pure GVBS states $\omega_{\alpha}, \alpha=1, \ldots, n$ and real numbers $t_{\alpha}>0, \sum_{\alpha} t_{\alpha}=1$, such that $\omega=\sum_{\alpha} t_{\alpha} \omega_{\alpha}$. For a GVBS state $\omega$ we will denote by $\mathscr{F}_{\omega}$ the set of all convex combinations of the states $\omega_{\alpha}$ that make up $\omega: \mathscr{F}_{\omega}=\left\{\eta=\sum_{\alpha} s_{\alpha} \omega_{\gamma} \mid s_{\alpha} \geqq 0, \sum_{\alpha} s_{\gamma}=1\right\}$. In other words $\mathscr{F}_{\omega}$ is the smallest set of states of the chain which contains $\omega$ and has the property: for any three states $\eta, \eta_{1}, \eta_{2}$ of the chain, if there exists a $t \in(0,1)$ such that $\eta=t \eta_{1}+(1-t) \eta_{2}$, then $\eta \in \mathscr{F}_{\omega} \Leftrightarrow \eta_{1}$ and $\eta_{2} \in \mathscr{F}_{\omega}$.

It is also known that for any finite set of GVBS states $\omega_{\alpha}$, any convex combination $\omega=\sum_{\alpha} t_{\alpha} \omega_{\alpha}$ is again GVBS. For a proof of these facts we refer to [14] and [70].

Above we were considering states $\omega$ of the infinite chain. They are in one-toone correspondence with a family of density matrices, one for each finite piece of the chain. We will denote by $\rho_{[M, N]}$ the density matrix in $\left(\mathscr{M}_{d}\right)^{\otimes(N-M+1)}$ such that $\omega(A)=\operatorname{tr} \rho_{[M, N]} A$ for all observables of the interval $[M, N]$, i.e. linear combinations of tensor products $A_{M} \otimes \cdots \otimes A_{N}$. The subspace of $\mathbb{C}^{\otimes(N-M+1)}$ spanned by the eigenvectors of $\rho_{[M, N]}$ belonging to the strictly positive eigenvalues, will be denoted by $\mathscr{G}_{[M, N]}$ and will be called the local support spaces of $\omega$. Obviously, $\mathscr{G}_{[M, N]}=$ $\rho_{[M, N]} \mathscr{H}_{[M, N]}$.

The main body of this paper is devoted to the proof of the following two theorems.

1. Theorem (Existence of GVBS Hamiltonians). For any GVBS state $\omega$ there exists a finite range interaction $h$, say of interaction length $l$, i.e. $0 \leqq h \in\left(\mathscr{M}_{d}\right)^{\otimes l}$, such that $\widetilde{\mathscr{F}}_{h}=\widetilde{\mathscr{F}}_{(1)}$ and for all intervals $[M, N]$ such that $N-M \geqq l$, one has 
$\operatorname{ker} H_{[M, N]}=\mathscr{G}_{[M, N]}$, where $H_{[M, N]}=\sum_{i=M}^{N-l+1} h_{i}$ and the $\mathscr{G}_{[M, N]}$ are the local support spaces of $\omega$.

In particular we can take for $\omega$ a convex combination of any finite set $\omega_{1}, \ldots, \omega_{n}$ of pure GVBS states. Theorem 1 then says that there exists a finite range Hamiltonian such that the set of infinite volume ground states of the Hamiltonian exactly coincides with the set of all convex combinations of the pure states $\omega_{1}, \ldots, \omega_{n}$. As the set of all pure translation invariant GVBS states is *-weakly dense in the set of all translation invariant states [69], Theorem 1 implies that any possible local behaviour can be approximated arbitrarily well by a GVBS model. In particular we can construct GVBS models with any possible kind of discrete symmetry breaking (see the discussion in Sect. 5).

2. Theorem (Existence of a Spectral Gap). Let $h$ be a finite range interaction such that there exists a GVBS state $\omega$ with the property that $\mathscr{F}_{\omega}=\mathscr{F}_{h}$, and such that $\operatorname{ker} H_{[M, N]}=\mathscr{G}_{[M, N]}$ for all integers $M$ and $N$ such that $N-M$ is large enough. Then there exists a constant $\gamma>0$ such that for all intervals $[M, N] \subset \mathbb{Z}$ one has that the second lowest eigenvalue of $H_{[M, N]}$ is at least $\gamma$ (the lowest eigenvalue being 0$)$. Moreover for any pure state $\eta \in \mathscr{F}_{\omega}$, and any local observable $X$ such that $\eta(X)=0$, one has

$$
\lim _{M, N \rightarrow \pm \infty} \eta\left(X^{*}\left[H_{[M, N]}, X\right]\right) \geqq \gamma \eta\left(X^{*} X\right) .
$$

For an explicit value of $\gamma$, i.e., a lower bound on the gap, see Sect. 6 .

It is important to note that the property $\mathscr{F}_{h}=\mathscr{F}_{\omega}$ does in general not imply that $\operatorname{ker} H_{[M, N]}=\mathscr{G}_{[M, N]}$ for all integers $M$ and $N$ such that $N-M$ is large enough. Indeed sometimes the latter property is absent and then there might be no gap directly above the ground state in the infinite volume model (see Sect. 7, Example 3).

From the technical point of view the crucial new ingredient needed for the proof of both Theorem 1 and Theorem 2 is Theorem 5, which proves the intersection property of the local support spaces of GVBS states. In [14] this property was proved for pure GVBS states. The extension to general GVBS states turns out to be rather nontrivial. The main element that was missing until now is the strong disjointness property of distinct pure GVBS states given in Lemma 6.

\section{Lower Bounds for the Spectral Gap - A General Strategy}

In this section we present in the form of a simple theorem a general strategy to obtain lower bounds for the gap in the spectrum of the generator of a class of spin dynamics. The basic argument, or ideas similar to it, has been used for analyzing irreversible time evolutions of classical spin systems [30-33] as well as in the study of the gap above the ground state of quantum spin Hamiltonians [12, 14]. Theorem 3 below is essentially an elaboration of a discussion with H.-T. Yau, who explained to the author his work with S.-L. Lu on the spectral gap in the generator of the Glauber and Kawasaki dynamics for Ising models.

The importance of having good estimates for the spectral gap is obvious: In the classical case the gap determines the speed with which the dynamics (see [35] for a precise statement) drives the system toward equilibrium. For quantum systems the essential features of the low-temperature physics are determined by the low-lying 
energy spectrum, in particular the gap between the ground state energy and the first excited state.

The general strategy, as it is described below, works only for models where the local terms in the generator of the dynamics are minimized individually in the reference state (see condition C2). For many quantum spin Hamiltonians this condition is not satisfied. We might hope, however, that once the existence of a spectral gap has been established for special models, perturbative methods could be developed (for some first steps in this direction see [74-78], which would enable one to show the existence of a spectral gap for a much wider class of models.

We have in mind the usual setup where a translation invariant model is defined by a net of local Hamiltonians $H_{\Lambda}$ indexed by finite volumes $\Lambda \subset \mathbb{Z}^{d}$, acting on Hilbert spaces $\mathscr{H}_{\Lambda}$. As we are interested in the behaviour of the spectrum in the thermodynamic limit, we introduce an increasing sequence of finite volumes $\left\{\Lambda_{n}\right\}_{n \in \mathbb{N}}$, such that any finite volume is eventually contained in the $\Lambda_{n}$, and with the convention that $\Lambda_{0}=\emptyset$. For the one-dimensional GVBS models, which are the main object of study in this paper, the typical choice for the $\Lambda_{n}$ would be an increasing sequence of intervals of the form $[1, p n]$ for some fixed integer $p$. We will always assume that local Hamiltonians $H_{\Lambda}$ are defined at least for all volumes of the form $\Lambda_{n} \backslash \Lambda_{m}$ for all $m \leqq n$. Typically they are given by

$$
H_{\Lambda}=\sum_{x, S+x \subset A} h_{x}
$$

where $h_{x}$ denotes the translate over $x \in \mathbb{Z}^{d}$ of an self-adjoint interaction operator $h$ acting on $\mathscr{H}_{S}$. For a one-dimensional model with a nearest neighbour interaction, $S$ consists of just two sites, say 0 and 1 .

For the theorem of this section only three conditions are needed. These conditions are stated as C1-3 below. They do not explicitly refer to the one-dimensionality or even translation invariance of the models. But, as was mentioned before, one should expect their verification for some suitable sequence $\Lambda_{n}$, in particular of condition $\mathrm{C} 3$, to be highly non-trivial in general. We will formulate the conditions first and then discuss their importance. In the case of GVBS models conditions $\mathrm{C} 1$ and $\mathrm{C} 2$ are automatically satisfied and for GVBS models with a unique ground state the proof of the C3 is contained in previous work [14].

Each of the assumptions involves some finite length $l$. We will assume that C1-C3 hold for one and the same $l \geqq 1$.

C1 There is a constant $d_{l}$ for which the local Hamiltonians satisfy:

$$
0 \leqq \sum_{n=l}^{N} H_{\Lambda_{n} \backslash \Lambda_{n-1}} \leqq d_{l} H_{\Lambda_{N}} .
$$

Often there will be an integer $r$ - which we can interpret as a measure of the range of the interaction - such that for all $l \geqq r$ and $N$ large enough, there is a constant $d_{l}$ for which $(2.1)$ holds. For a translation invariant one-dimensional system with an interaction of range $r$ and $\Lambda_{n}=[1, n]$, one could simply take $d_{l}=l-r+1$. Sometimes it is convenient to choose $\Lambda_{n}=[1, p n]$, with $p \geqq 2$. If $p \geqq r$ one can take $d_{l}=l$.

C2 We assume that there is a non-trivial subspace $\mathscr{G}_{\Lambda_{n}}$ of $\mathscr{H}_{\Lambda_{n}}$ consisting of all vectors $\psi$ such that $H_{\Lambda_{n}} \psi=0$. For any finite volume $\Lambda \subset \Lambda_{N}$ we denote by $G_{\Lambda}$ the 
orthogonal projection onto the space $\mathscr{G}_{\Lambda} \otimes \mathscr{H}_{\Lambda_{N} \backslash \Lambda}$. The local Hamiltonians have a non-vanishing spectral gap $\gamma_{l}>0$ :

$$
H_{\Lambda_{n} \backslash \Lambda_{n-1}} \geqq \gamma_{l}\left(\mathbb{1}-G_{\Lambda_{n} \backslash \Lambda_{n-1}}\right) \text { for all } n \geqq n_{l},
$$

where $n_{l}$ is some appropriate constant. In the case of one-dimensional systems with interactions of a finite range $r$ one could take $n_{l}=l \geqq r$.

By convention we put $G_{\Lambda_{0}}=G_{\emptyset}=\mathbb{1}$, and $G_{\Lambda_{N+1}}=0$. Note that $G_{\Lambda}$ and $G_{\Lambda^{\prime}}$ commute if either $\Lambda^{\prime} \subset \Lambda$ (in which case $G_{\Lambda^{\prime}}=G_{\Lambda^{\prime}} G_{\Lambda}=G_{\Lambda} G_{\Lambda^{\prime}}$ ) or $\Lambda^{\prime} \cap \Lambda=\emptyset$ (in which case $G_{\Lambda^{\prime}} G_{\Lambda}=G_{\Lambda \cup \Lambda^{\prime}}$ ). It follows that the operators $E_{n}, n=0, \ldots, N$, defined by

$$
E_{n}=G_{\Lambda_{n}}-G_{\Lambda_{n+1}}
$$

form a complete family of mutually orthogonal projections, i.e., $\sum_{n=0}^{N} E_{n}=\mathbb{1}$ and $E_{n} E_{m}=\delta_{n, m} E_{n}$.

The third condition is the crucial one in the present context. We present two versions of it, $\mathrm{C} 3$ and $\mathrm{C}^{\prime}$. The conditions $\mathrm{C} 1-3$ are sufficient for the existence of a uniform lower bound on the spectral gaps of the local Hamiltonians, but when $\mathrm{C}^{\prime}$ holds better explicit estimates for the spectral gap can be obtained. In the latter case $\mathrm{C} 1$ should also be replaced by $\mathrm{Cl}^{\prime}$ stated below.

C3 We assume that there exist $\varepsilon_{l}<1 / \sqrt{l}$ and an integer $n_{l}$ such that

$$
\left\|G_{\Lambda_{n+1} \backslash \Lambda_{n+1-l}} E_{n}\right\| \leqq \varepsilon_{l} \text { for all } n, n_{l} \leqq n \leqq N-1
$$

or equivalently

$$
E_{n} G_{\Lambda_{n+1} \backslash \Lambda_{n+1-l}} E_{n} \leqq \varepsilon_{l}^{2} E_{n}
$$

C3' There exist constants $n_{l}$, and $\eta_{l}<1 / \sqrt{2}$ such that

$$
\left\|G_{\Lambda_{n+p} \backslash \Lambda_{n+1-l}} E_{n}^{(p)}\right\| \leqq \eta_{l} \quad \text { for all } 1 \leqq p \leqq l, n \geqq n_{l} \text { and } l \geqq l_{0}
$$

where $E_{n}^{(p)}=\sum_{k=n}^{n+p-1} E_{k}$.

C1' There is an integer $r$ - which we can interpret as a measure of the range of the interaction - and a constant $d$ such that for all $l \geqq r$ and $N=l M$ large enough, the local Hamiltonians satisfy:

$$
0 \leqq \sum_{m=1}^{M} H_{\Lambda_{l m} \backslash \Lambda_{l(m-1)}} \leqq d H_{\Lambda_{N}}
$$

For a translation invariant one-dimensional system with an interaction of range $r$ one can simply take $d=2$.

\section{Theorem.}

i) Assume that the conditions $C 1-3$ are satisfied for one and the same integer $l$. Then, for any $N$ and any $\psi \in \mathscr{H}_{\Lambda_{N}}$ such that $G_{\Lambda_{N}} \psi=0$, i.e., $\psi$ is orthogonal to the space of ground states of $H_{\Lambda_{N}}$, one has

$$
\left\langle\psi \mid H_{\Lambda_{N}} \psi\right\rangle \geqq \frac{\gamma_{l}}{d_{l}}\left(1-\varepsilon_{l} \sqrt{l}\right)^{2}\|\psi\|^{2} .
$$


ii) If $C 1^{\prime}, C 2$, and $C 3^{\prime}$ hold, then for all $N=l M$,

$$
\left\langle\psi \mid H_{\Lambda_{N}} \psi\right\rangle \geqq \frac{\gamma_{2 l}}{d}\left(1-\sqrt{2} \eta_{l}\right)^{2}\|\psi\|^{2} .
$$

The proof of this theorem is rather elementary. Of course all essential information is hidden in the conditions $\mathrm{C} 1-3$.

Condition $\mathrm{C} 1$ is a simple assumption on the (quasi-) local structure of the Hamiltonians and the structure of the sequence $\Lambda_{n}$. It is trivial for one-dimensional systems and $\Lambda_{n}$ which are intervals increasing in a regular way.

Condition C2 restricts the applicability of the method to models where the energy is minimized locally. It is a non-frustration condition (see $[45,73]$ for a discussion). For quantum spin models this is the case for "purely ferromagnetic" interactions (but then, as is shown below, $\mathrm{C} 3$ is not satisfied uniformly in $N$ because of the breaking of the continuous rotation symmetry), and the models of the Valence-Bond-Solid type studied in $[12,14]$. It is an interesting open problem to prove the existence of a gap under weaker versions of $\mathrm{C} 2$, e.g. where one controls the corrections to local energy minimization.

The hard work is to check Condition $\mathrm{C} 3$ or $\mathrm{C} 3^{\prime} . \mathrm{C} 3$ plays the role of a mixing condition similar to the Dobrushin-Shlosman condition for ergodicity. It is a wellknown fact for the conditional expectations in the Gibbs state of a one-dimensional classical spin system with finite range interactions, and one would expect it to be generally true also for the ground states of quantum chains under the assumption that there is sufficient (exponential) decay of spatial correlations.

The operators $E_{n}$ defined in (2) are "conditional expectations" in the ground state. In a model where the energy is not minimized locally, one could still define the $E_{n}$ using the local restrictions of an infinite volume limit of the local ground states. But then, generically, C2 cannot be expected to be satisfied. When studying a stochastic dynamics for a classical spin model, one would define them to be conditional expectations in the equilibrium state (see e.g. [32]).

Note that $G_{\Lambda_{n+1} \backslash \Lambda_{n+1-1}} E_{n}=G_{\Lambda_{n+1} \backslash \Lambda_{n+1-1}} G_{\Lambda_{n}}-G_{\Lambda_{n+1}}$. In the case of pure GVBS states, norm bounds on this quantity are available from [14], where it is shown that there exist constants $c \geqq 0$ and $0 \leqq \lambda<1$ such that

$$
\left\|G_{\Lambda_{n}} G_{\Lambda_{n+1} \backslash \Lambda_{n+1-1}}-G_{\Lambda_{n+1}}\right\| \leqq c \lambda^{l} \frac{1+c \lambda^{l}}{1-c \lambda^{l}} .
$$

In this paper the main effort of proving the existence of a spectral gap consists in showing that (2.5) holds with an $\varepsilon_{l}<1 / \sqrt{l}$ for all large enough $n$ (Sect. 6).

\section{Proof of Theorem 3}

i) From the definition of the $E_{n}(2.3)$ and the assumption that $G_{\Lambda_{N}} \psi=0$, it immediately follows that

$$
\psi=\sum_{n=0}^{N-1} E_{n} \psi,
$$

and the fact that the $E_{n}$ are mutually orthogonal projections implies that

$$
\|\psi\|^{2}=\sum_{n=0}^{N-1}\left\|E_{n} \psi\right\|^{2}
$$


Define $G_{n, l}=G_{\Lambda_{n+1} \backslash \Lambda_{n+1-1}}$ for $n \geqq n_{l}$ and for convenience put $G_{n, l}=0$ for $n<n_{l}$.

Due to (2.7) one has the identity

$$
\begin{aligned}
\left\|E_{n} \psi\right\|^{2} & =\left\langle\psi \mid\left(\mathbb{1}-G_{n, l}\right) E_{n} \psi\right\rangle+\left\langle\psi \mid G_{n, l} E_{n} \psi\right\rangle \\
& =\left\langle\psi \mid\left(\mathbb{1}-G_{n, l}\right) E_{n} \psi\right\rangle+\left\langle\psi \mid \sum_{m=0}^{N-1} E_{m} G_{n, l} E_{n} \psi\right\rangle .
\end{aligned}
$$

Because $G_{\Lambda}$ and $G_{\Lambda^{\prime}}$ commute if either $\Lambda^{\prime} \subset \Lambda$ or $\Lambda^{\prime} \cap \Lambda=\emptyset$, also $E_{m}$ commutes with $G_{n, l}$ if either $m \leqq n-l$ or $m \geqq n+1$. In these cases $E_{m} G_{n, l} E_{n}=G_{n, l} E_{m} E_{n}=$ 0 , because the $E_{n}$ form an orthogonal family. Using this observation we obtain the following estimate from (2.9). For any choice of constants $c_{1}, c_{2}>0$ :

$$
\begin{aligned}
\left\|E_{n} \psi\right\|^{2}= & \left\langle\psi \mid\left(\mathbb{1}-G_{n, l}\right) E_{n} \psi\right\rangle+\left\langle\sum_{m=n+1-l}^{n} E_{m} \psi \mid G_{n, l} E_{n} \psi\right\rangle \\
\leqq & \frac{1}{2} c_{1}\left\langle\psi \mid\left(\mathbb{1}-G_{n, l}\right) \psi\right\rangle+\frac{c_{1}}{2}\left\langle\psi \mid E_{n} \psi\right\rangle+\frac{1}{2 c_{2}}\left\langle\psi \mid E_{n} G_{n, l} E_{n} \psi\right\rangle \\
& +\frac{c_{2}}{2}\left\langle\psi \mid\left(\sum_{m=n+1-l}^{n} E_{m}\right)^{2} \psi\right\rangle,
\end{aligned}
$$

where we have applied the inequality

$$
\left|\left\langle\varphi_{1} \mid \varphi_{2}\right\rangle\right| \leqq \frac{1}{2 c}\left\|\varphi_{1}\right\|^{2}+\frac{c}{2}\left\|\varphi_{2}\right\|^{2}
$$

for any $c>0$, to both terms. The first term in the right side of inequality $(2.10)$ can be estimated with the Hamiltonian due to condition C2 (2.2). To the third term we apply condition C3 (2.4). It then follows that

$$
\left(2-c_{1}-\frac{\varepsilon_{l}^{2}}{c_{2}}\right)\left\|E_{n} \psi\right\|^{2}-c_{2} \sum_{m=n+1-l}^{n}\left\|E_{m} \psi\right\|^{2} \leqq \frac{1}{c_{1} \gamma_{l}}\left\langle\psi \mid H_{\Lambda_{n+1} \backslash \Lambda_{n+1-l}} \psi\right\rangle .
$$

We now sum over $n$, use (2.8), and apply condition C1 (2.1) to obtain

$$
\left(2-c_{1}-\frac{\varepsilon_{l}^{2}}{c_{2}}-l c_{2}\right)\|\psi\|^{2} \leqq \frac{d_{l}}{c_{1} \gamma_{l}}\left\langle\psi \mid H_{\Lambda_{N}} \psi\right\rangle \text {. }
$$

Finally put $c_{1}=1-\varepsilon_{l} \sqrt{l}$ and $c_{2}=\varepsilon_{l} / \sqrt{l}$ and one obtains the estimate i) stated in the theorem.

ii) In order to obtain the improved estimate under condition $\mathrm{C}^{\prime}$ (2.5), one just applies i) of above with $l=2$ and with a "rescaled" increasing sequence of finite volumes $\tilde{\Lambda}_{n}$, defined by

$$
\tilde{\Lambda}_{n}=\Lambda_{\text {ln }}
$$

and by using $\tilde{G}_{n, l}=G_{\tilde{\Lambda}_{n+1} \backslash \tilde{\Lambda}_{n-1}}$ instead of $G_{n, l}$, and the obvious relations $\tilde{\eta}_{k}=\varepsilon_{l k}$, $\tilde{\gamma}_{k}=\gamma_{l k}$.

For the GVBS models it is easy to show (see the proof of Proposition 10 in Sect. 6) that a uniform lower bound on the spectral gap of the finite-volume Hamiltonians implies the existence of spectral gap - bounded from below by the same 
lower bound - in the thermodynamic limit, i.e., in the spectrum of the Hamiltonian in the GNS representation of one of the finitely many pure infinite-volume ground states. The same relation holds for any model with finite range interactions and for which the infinite volume ground states can be obtained as limits of finite volume pure ground states. The proof of Theorem 2 is therefore reduced to showing that the conditions $\mathrm{C} 1-3^{\prime}$ hold for the GVBS models under consideration.

We conclude this section with some remarks on the quality of the lower bounds for the gap that are obtained in Theorem 2. At the same time we will illustrate with an example that one can also use these estimates in situations where there is no gap in the thermodynamic limit. The simplest example of this situation is the spin-1/2 Heisenberg ferromagnetic chain. In this case Theorem 2 still gives a lower bound for the finite volume gaps which is the correct order of magnitude as a function of the size of the finite system.

The Hamiltonian of the spin-1/2 Heisenberg ferromagnetic chain of length $N+1$, which acts on $\left(\mathbb{C}^{2}\right)^{\otimes(N+1)}$, can be written as follows:

$$
H_{N}=\sum_{i=1}^{N} \frac{1}{2}\left(\mathbb{1}-T_{i, i+1}\right),
$$

where $T_{l, i+1}$ is the permutation operator that interchanges the states at sites $i$ and $i+1$. From this formula for the Hamiltonian it immediately follows that the ground state projection for an interval $[a, b], G_{[a, b]}$, is the orthogonal projection onto the space of permutation symmetric states. For $M=1, \ldots, N$, define $\varepsilon_{2}^{(N)}$ by

$$
\varepsilon_{2}^{(M)}=\left\|G_{[1, M]} G_{[M, M+1]}-G_{[1, M+1]}\right\|=\left\|G_{[M, M+1]} G_{[1, M]}-G_{[1, M+1]}\right\| .
$$

Theorem 2 i) and the remarks above imply that the gap $\gamma_{N}$ of $H_{N}$ satisfies

$$
\gamma_{N} \geqq \gamma_{2}\left(1-\varepsilon_{2} \sqrt{2}\right)^{2},
$$

where

$$
\varepsilon_{2}=\sup _{1 \leqq M \leqq N} \varepsilon_{2}^{(M)} .
$$

$\gamma_{2}$ is the gap of $\frac{1}{2}\left(\mathbb{1}-T_{i, i+1}\right)$ which is 1 . A straightforward spin-wave upper bound for the gap for large $N$ is $\gamma_{N} \leqq$ Constant/ $N^{2}$. The bound (2.11) yields non-trivial information only if $\varepsilon_{2}<1 / \sqrt{2}$. On the other hand any $\varepsilon_{2}<1 / \sqrt{2}$ uniform in $N$ would imply the existence of a spectral gap in the thermodynamic limit. The following lemma shows that, in general, this critical value of $\varepsilon_{2}=1 / \sqrt{2}$ is optimal.

4. Lemma. For the spin-1/2 Heisenberg ferromagnetic chain on an interval of length $N+1, N \geqq 2$, we have

$$
\varepsilon_{2}=\frac{1}{\sqrt{2}} \sqrt{1-\frac{1}{N}} .
$$

Proof. Observe that $G_{[M, M+1]} G_{[1, M]}$ and $G_{[1, M+1]}$ commute and that

$$
G_{[M, M+1]} G_{[1, M]} G_{[1, M+1]}=G_{[1, M+1]} .
$$

$\varepsilon_{2}^{(M)}$ can therefore be computed as

$$
\sup _{\psi} \frac{\left\|G_{[M, M+1]} G_{[1, M]} \psi\right\|}{\|\psi\|},
$$


where the sup is taken over $0 \neq \psi$ such that $G_{[1, M+1]} \psi=0$. Obviously it is sufficient to consider $\psi$ satisfying $G_{[1, M]} \psi=\psi$. Due to the $S U(2)$ invariance of all operators we can conclude that the sup must be attained for the vector

$$
\psi=\sum_{x=1}^{M} S_{x, M+1}=M D_{M+1}-\sum_{x=1}^{M} D_{x},
$$

where $S_{x, y}=D_{y}-D_{x}$ and $D_{z}=S_{z}^{-} \mid$all up $\rangle$, for $z=1, \ldots, M+1$. We then just have to compute $G_{[M, M+1]} \psi$ :

$$
\varphi \equiv G_{[M, M+1]} \psi=\frac{1}{2}(M-1)\left(D_{M}+D_{M+1}\right)-\sum_{x=1}^{M-1} D_{x} .
$$

It is then trivial to verify that $\|\psi\|^{2}=M^{2}+M$ and $\|\varphi\|^{2}=(M-1)^{2} / 2+(M-1)$. Hence

$$
\varepsilon_{2}^{(M)}=\sqrt{\frac{\|\varphi\|^{2}}{\|\psi\|^{2}}}=\frac{1}{\sqrt{2}} \sqrt{\frac{M-1}{M}} .
$$

As $\varepsilon_{2}^{(M)}$ is monotone increasing in $M$ its supremum, $\varepsilon_{2}$, is attained in $M=N$.

Note that the gap estimate (2.11) for finite volumes is of the same order in $N$ as the upper bound from spin waves. Estimates of the number of low energy states in the Heisenberg and other models are given in [79]. In Sect. 6 we will compare the lower bounds on the spectral gap of GVBS models that follow from Theorem 3 with previous work on GVBS models.

\section{Some Basic Facts on Pure GVBS States}

Here we collect the basic properties of pure GVBS states that we will need in the sequel. Proofs can be found in [14]. For a review on GVBS states see [80].

Throughout this section $\omega$ is a pure, translation invariant state of the infinite chain. Let $\mathscr{H}_{i} \cong \mathbb{C}^{d}$ denote the Hilbert space at a site $i \in \mathbb{Z}$, and for any finite subset $\Lambda \subset \mathbb{Z}$, we define $\mathscr{H}_{\Lambda}=\bigotimes_{i \in \Lambda} \mathscr{H}_{l}$. In particular, for $M \leqq N \in \mathbb{Z}, \mathscr{H}_{[M, N]}$ denotes the state space of a finite piece of the chain of length $N-M+1$. For convenience we put $\mathscr{H}_{\emptyset}=\mathbb{C}$, and for any Hilbert space $\mathscr{H}$, we will identify $\mathbb{C} \otimes \mathscr{H}$ and $\mathscr{H} \otimes \mathbb{C}$ with $\mathscr{H}$ itself. Let $\mathscr{M}_{d}$ denote the complex $d \times d$ matrices.

Suppose $\omega$ is a state obtained by the GVBS construction as outlined in Sect. 1, i.e. there is a $k \geqq 1$, a linear map $W: \mathbb{C}^{d} \rightarrow \mathbb{C}^{k} \otimes \mathbb{C}^{k}$, and a vector $\varphi \in \mathbb{C}^{k} \otimes \mathbb{C}^{k}$ such that

$$
\begin{aligned}
& \omega\left(A_{1} \otimes \cdots \otimes A_{n}\right) \\
& =\lim _{\substack{M \rightarrow-\infty \\
N \rightarrow+\infty}} \frac{\left\langle\varphi^{\otimes N-M+2}\left|P_{M, N} \otimes W \mathbb{1}_{d} W^{*} \otimes \cdots W A_{1} W^{*} \cdots W A_{n} W^{*} \cdots \otimes Q_{M, N}\right| \varphi^{\otimes N-M+2}\right\rangle}{\mathscr{N}(M, N)},
\end{aligned}
$$

where $0 \leqq P_{M, N}, Q_{M, N} \in \mathscr{M}_{k}$ are chosen in one of the possible ways to obtain a well-defined limiting state $\omega$. It is then shown in [14] (Lemma 3.5 combined with 
Propositon 3.7), that without loss of generality we can assume that the following equations are satisfied:

$$
\left(\mathrm{id}_{\mathscr{M}_{k}} \otimes \Phi\right)\left(W \mathbb{1}_{d} W^{*} \otimes \mathbb{1}_{k}\right)=\mathbb{1}_{k}
$$

and

$$
\left(\Phi \otimes \operatorname{id}_{\mathscr{H}_{k}}\right)\left(\mathbb{1}_{k} \otimes W \mathbb{1}_{d} W^{*}\right)=\mathbb{1}_{k},
$$

where $\Phi$ is the map $\mathscr{M}_{k} \otimes \mathscr{M}_{k} \rightarrow \mathbb{C}$ defined by $\Phi(X)=\langle\varphi \mid X \varphi\rangle$ and id $\mathscr{M}_{k}$ denotes the identity map of $\mathscr{M}_{k}$, i.e. id $\mathscr{M}_{k}(X)=X$. This means that for a given state $\omega$ we can redefine our objects, such that $\mathscr{N}=1$ and $P_{M, N}=Q_{M, N}=\mathbb{1}_{k}$ and such that moreover the limit in (3.1) becomes redundant: one can take $[M, N]=[1, n]$ to calculate the correct expectation value of $A_{1} \otimes \cdots \otimes A_{n}$ in the thermodynamic limit:

$$
\omega\left(A_{1} \otimes \cdots \otimes A_{n}\right)=\left\langle\varphi^{\otimes n+1}\left|\mathbb{1}_{k} \otimes W A_{1} W^{*} \otimes \cdots \otimes W A_{n} W^{*} \otimes \mathbb{1}_{k}\right| \varphi^{\otimes n+1}\right\rangle .
$$

It is also useful to define for all $A \in \mathscr{M}_{d}$ the operator $\mathbb{E}_{A}: \mathscr{M}_{k} \rightarrow \mathscr{M}_{k}$ by:

$$
\mathbb{E}_{A}(B)=\left(\mathrm{id}_{\mathscr{M}_{k}} \otimes \Phi\right)\left(W A W^{*} \otimes B\right),
$$

and a state $\rho$ of $\mathscr{M}_{k}$ by: $\rho(B)=\left\langle\varphi \mid \mathbb{1}_{k} \otimes B \varphi\right\rangle$. (3.3) then becomes

$$
\omega\left(A_{1} \otimes \cdots \otimes A_{n}\right)=\rho\left(\mathbb{E}_{A_{1}} \circ \cdots \circ \mathbb{E}_{A_{n}}\left(\mathbb{1}_{k}\right)\right) .
$$

Instead of (3.3) we can as well write:

$$
\mathbb{E}_{A}(B)=V^{*} A \otimes B V
$$

with $V: \mathbb{C}^{k} \rightarrow \mathbb{C}^{d} \otimes \mathbb{C}^{k}$ another isometry. It is obvious that many choices of $k, W$ and $\varphi$, even under the restrictive conditions (3.2), will lead to the same state $\omega$. In particular, if $\omega$ can be constructed with some $W: \mathbb{C}^{d} \rightarrow \mathbb{C}^{k} \otimes \mathbb{C}^{k}$, then, by a trivial extension of the maps, we will also have representations of $\omega$ with maps $W^{\prime}: \mathbb{C}^{d} \rightarrow \mathbb{C}^{k^{\prime}} \otimes \mathbb{C}^{k^{\prime}}$ with $k^{\prime}>k$. A possible way to express that the dimension $k$ is as small as possible for a given GVBS state, is the following. Consider the subalgebra $\mathscr{B}$ of $\mathscr{M}_{k}$, generated by the elements of the form $\mathbb{E}_{A_{1}} \circ \cdots \circ \mathbb{E}_{A_{n}}\left(\mathbb{1}_{k}\right)$, where $n \geqq 1$, and $A_{1}, \ldots, A_{n} \in \mathscr{M}_{d}$. Then let $k_{0}$ be the smallest integer such that $\mathscr{B}$ can be faithfully represented as a subalgebra of $\mathscr{M}_{k_{0}}$. The minimality condition we need is $k=k_{0}$. Under this condition it can be shown that the objects that appear in the GVBS construction are uniquely determined by the state $\omega$ up to unitary equivalence ([14], Theorem 1.3).

The states obtained by (3.1) are not necessarily pure, i.e. they may have nontrivial decompositions into other states. A very tractable characterization of the purity of $\omega$, is given in terms of the transition operator $\mathbb{P} \equiv \mathbb{E}_{\mathbb{1}}$. $\mathbb{P}$ is a completely positive transformation of $\mathscr{M}_{k}$, and (3.2a) just says that $\mathbb{P}\left(\mathbb{1}_{k}\right)=\mathbb{1}_{k}$. These two properties make $\mathbb{P}$ a Markov operator (i.e. $\mathbb{P}$ is the straightforward generalization of a Markov operator to the non-abelian context [67]). $\mathbb{P}$ governs the ergodic properties of the state $\omega$. It turns out that under the minimality condition stated above $\left(k=k_{0}\right), \omega$ is pure iff $\mathbb{P}(X)=\lambda X$ with $|\lambda|=1 \Rightarrow \lambda=1$ and $X$ a multiple of $\mathbb{1}_{k}$ : i.e. the peripheral spectrum of $\mathbb{P}$ consists only of the non-degenerate eigenvalue 1 (for a proof of the if-part see [14], Propositon 5.9; for the only-if part see [70], Theorem 1.4). So we have a very simple criterion that tells us exactly when $\omega$ is a pure state. For pure GVBS states very detailed results are obtained in [14]. In particular it follows that 
there always exists a translation invariant finite range interaction such that $\omega$ is the unique ground state of the corresponding model and such that the Hamiltonian has a spectral gap above the ground state. Let us go step by step and list the essential properties of pure GVBS states that will be used in the following sections with the aim to extend essentially these same properties to GVBS states that are not necessarily pure.

Let again $\rho_{[M, N]}$ be the density matrix describing the pure state $\omega$ restricted to the interval $[M, N]$. Then there are $k$ real numbers, $\rho_{1}>0, \ldots, \rho_{k}>0$, such that the non-vanishing spectrum of $\rho_{[M, N]}$ is asymptotically equal to $\left\{\rho_{l} \rho_{j} \mid 1 \leqq i, j \leqq k\right\}$ in the limit $N-M \rightarrow \infty$. The relevant property which follows from this observation is that

$$
\inf _{M \leqq N}\left(\operatorname{spec}\left(\rho_{[M, N]}\right) \backslash\{0\}\right)>0 \text {. }
$$

Define

$$
m_{0}=\inf \left\{m \geqq 1 \mid \operatorname{dim} \mathscr{G}_{[1, m]}=k^{2}\right\},
$$

then one can show that $\operatorname{dim} \mathscr{G}_{[M, N]}=k^{2}$ for all $M, N$ such that $N-M \geqq m_{0}-1$. For $N-M \geqq m_{0}-1$, let $\psi_{l, j}^{M, N}$ denote a set of normalized eigenvectors of $\rho_{[M, N]}$, belonging to the non-zero eigenvalues. For any local observable $A$ we then have:

$$
\lim _{M, N \rightarrow \pm \infty}\left\langle\psi_{i, J}^{M, N} \mid A \psi_{k, l}^{M, N}\right\rangle=\omega(A) \delta_{i, k} \delta_{j, l}
$$

One can also show that if $\omega$ and $\eta$ are two different pure GVBS states of the same chain, and with local support vectors $\psi_{l, j}^{M, N}$ and $\chi_{i, j}^{M, N}$ respectively, then for any local observable $A$ :

$$
\lim _{M, N \rightarrow \pm \infty}\left\langle\psi_{i, j}^{M, N} \mid A \chi_{k, l}^{M, N}\right\rangle=0 .
$$

We will give a proof of this property in Sect. 4 (Lemma 6). The orthogonal projection onto the subspace $\mathscr{G}_{[M, N]}$ of $\mathscr{H}_{[M, N]}$ spanned by the vectors $\psi_{l, j}^{M, N}$ will be denoted by $G_{[M, N]}$. The spaces $\mathscr{G}_{[M, N]}$ satisfy a nice intersection property: there exists an integer $l_{0} \geqq 1$ such that for all $l \geqq l_{0}$ and all $M, N \in \mathbb{Z}$ such that $N-M \geqq l$ one has

$$
\mathscr{G}_{[M, N]}=\bigcap_{k=0}^{N-M-l+1} \mathscr{H}_{[M, M+k-1]} \otimes \mathscr{G}_{[M+k, M+k+l-1]} \otimes \mathscr{H}_{[M+k+l, N]} .
$$

Here $l_{0}$ can always be taken to be equal to $m_{0}+1$, with $m_{0}$ defined in (3.7). In some, but not all cases. (3.10) also holds with $l=m_{0}$. The following equivalent form of (3.10) is sometimes useful: for all $l \geqq l_{0}$, all $a, b, c \in \mathbb{Z}$ such that $a \leqq b, b+l \leqq c$, one has

$$
\mathscr{G}_{[a, c]}=\mathscr{G}_{[a, b+l]} \otimes \mathscr{H}_{[b+l+1, c]} \cap \mathscr{H}_{[a, b]} \otimes \mathscr{G}_{[b+1, c]} .
$$

The intersection property is closely related with the existence of finite range interactions for which the state $\omega$ is the unique ground state. Let $p \geqq 1$ be a regrouping parameter and take $l$ such that $p l \geqq l_{0}-1+p$. Let $h \in\left(\mathscr{M}_{d}\right)^{\overline{p l}}$ be a non-negative definite observable such that $\operatorname{ker} h=\mathscr{G}_{[1, p l]}$. Define local Hamiltonians for the regrouped chain by:

$$
H_{[p M, p N]}=\sum_{i=M}^{N-l+1} h_{p l}
$$


Then

$$
\operatorname{ker} H_{[p M, p N]}=G_{[p M, p N]}
$$

for all $M, N$ such that $N-M \geqq l$. This we call the ground state property of $\omega$. Moreover for two such Hamiltonians $H$ and $H^{\prime}$, obtained by interactions $h$ and $h^{\prime}$ of ranges $p l$ and $p^{\prime} l^{\prime}$, there exist constants $C_{1}>0$ and $C_{2}$ such that for all intervals $[M, N], N-M$ large enough and compatible with the periodicity of the Hamiltonians:

$$
C_{1} H_{[M, N]} \leqq H_{[M, N]}^{\prime} \leqq C_{2} H_{[M, N]} .
$$

We will also need the following property of the ground state projections $G_{[M, N]}$ : there exists a $C>0$ and a $0 \leqq \lambda<1$ such that for all $l \geqq l_{0}, a, b, c \in \mathbb{Z}, a \leqq b$, $b+l \leqq c$, one has

$$
\left\|G_{[a, c]}-\left(G_{[a, b+l]} \otimes \mathbb{1}_{[b+l+1, c]}\right)\left(\mathbb{1}_{[a, b]} \otimes G_{[b+1, c]}\right)\right\| \leqq C \lambda^{l}
$$

We call this the commutation property of the ground state projections. Indeed (3.15) implies that the ground state projections for two intervals that have a large intersection, almost commute. This property is related with the "good factorization property" proved in [81] for GVBS states and similar to the factorization property for some classical partition functions, given in [82].

Finally, for any choice of $h$, the Hamiltonians $H_{[M, N]}$ defined in (3.12) have a non-vanishing spectral gap: there exists a constant $\gamma>0$ such that for all intervals $[M, N]$ :

$$
\left(H_{[M, N]}\right)^{2} \geqq \gamma H_{[M, N]} \geqq 0 .
$$

For GVBS models, (3.16) implies a gap of at least $\gamma$ in the spectrum of the GNSHamiltonian of the infinite system.

\section{The Intersection Property of GVBS States}

The aim of this section is to extend the intersection property (3.10), or equivalently (3.11), to arbitrary GVBS states, i.e. dropping the condition that they are pure. This property will be essential in the proof of Theorem 1 and Theorem 2 . We believe that under the condition that the dimension of the support spaces of the local restrictions of the ground states is bounded (or approximately bounded), the intersection property actually implies the existence of a spectral gap by itself, whether the ground states are VBS-like or not. In the next section we will prove that for a GVBS state the intersection property is equivalent with the existence of a finite range interaction giving rise to (3.13) (the ground state property).

5. Theorem (Intersection Property). Let $\omega_{1}, \ldots, \omega_{n}$ be $n$ distinct, pure GVBS states of a quantum spin chain. Then, the support spaces $\mathscr{G}_{\Lambda}$ of any state $\omega$ which is a convex combination of the $\omega_{1}, \ldots, \omega_{n}$, have the intersection property, i.e., there exists a constant $m_{0}$ such that for all $l, m, r$ satisfying $l \leqq 1, m \geqq m_{0}$, and $r \geqq m$ we have

$$
\mathscr{G}_{[l, r]}=\mathscr{G}_{[l, m]} \otimes \mathscr{H}_{[m+1, r]} \cap \mathscr{H}_{[l, 0]} \otimes \mathscr{G}_{[1, r]} .
$$


The proof of this theorem follows from the intersection property of pure GVBS states (3.10), an orthogonality property of pure GVBS states proved in Lemma 6, and Proposition 7. Proposition 7 itself does not involve the GVBS nature of the states directly. It is a purely geometric property of the support spaces.

In the considerations that follow the notion of overlap between Hilbert spaces will play a crucial role. For any two subspaces $\mathscr{H}_{0}$ and $\mathscr{H}_{1}$ of a Hilbert space $\mathscr{H}$, we define the overlap as follows:

$$
\mathcal{O}\left(\mathscr{H}_{0}, \mathscr{H}_{1}\right)=\sup _{\substack{0 \neq \varphi \in \mathscr{H}_{0} \\ 0 \neq \psi \in \mathscr{H}_{1}}} \frac{|\langle\varphi \mid \psi\rangle|}{\|\varphi\| \| \psi} .
$$

The overlap is the cosine of the angle between the subspaces. The following properties of the overlap are elementary:

i) $0 \leqq \mathcal{O}\left(\mathscr{H}_{0}, \mathscr{H}_{1}\right)=\mathcal{O}\left(\mathscr{H}_{1}, \mathscr{H}_{0}\right) \leqq 1$.

ii) $\mathcal{O}\left(\mathscr{H}_{0}, \mathscr{H}_{1}\right)<1$ if and only if $\mathscr{H}_{0} \cap \mathscr{H}_{1}=\{0\}$.

iii) $O\left(\mathscr{H}_{0}, \mathscr{H}_{1}\right)=0$ if $\mathscr{H}_{0} \perp \mathscr{H}_{1}$.

iv) If $\mathscr{H} \subset \mathscr{H}^{\prime}$, then the overlap remains unchanged if $\mathscr{H}_{0}$ and $\mathscr{H}_{1}$ are now considered as subspaces of $\mathscr{H}^{\prime}$ rather than of $\mathscr{H}$.

v) For any Hilbert space $\mathscr{K}$, consider the subspaces $\mathscr{H}_{0} \otimes \mathscr{K}$ and $\mathscr{H}_{1} \otimes \mathscr{K}$ of $\mathscr{H} \otimes \mathscr{K} ;$ again the overlap is unaffected: $\mathcal{O}\left(\mathscr{H}_{0} \otimes \mathscr{K}, \mathscr{H}_{1} \otimes \mathscr{K}\right)=\mathscr{O}\left(\mathscr{H}_{0}, \mathscr{H}_{1}\right)$. vi) If $\mathscr{H}_{0} \subset \mathscr{H}_{0}^{\prime} \subset \mathscr{H}$ and $\mathscr{H}_{1} \subset \mathscr{H}$, then $\mathcal{O}\left(\mathscr{H}_{0}, \mathscr{H}_{1}\right) \leqq \mathcal{O}\left(\mathscr{H}_{0}^{\prime}, \mathscr{H}_{1}\right)$.

Using these properties of the overlap and the specific properties of the local support spaces $\mathscr{G}_{[M, N]}^{\alpha}$ and $\mathscr{G}_{[M, N]}^{\beta}$ of two pure GVBS states of the same chain, say $\omega_{\alpha}$ and $\omega_{\beta}$, it is easy to show that for all $l, m, r \geqq 1$ :

$$
\mathcal{O}\left(\mathscr{G}_{[1, l+m]}^{\alpha} \otimes \mathscr{H}_{[l+m+1, l+m+r]}, \mathscr{H}_{[1, l]} \otimes \mathscr{G}_{[l+1, l+m+r]}^{\beta}\right) \leqq \mathcal{O}\left(\mathscr{G}_{[1, m]}^{\alpha}, \mathscr{G}_{[1, m]}^{\beta}\right),
$$

and from the next lemma it follows that

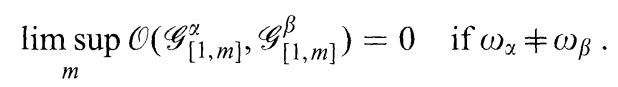

6. Lemma. Let $\omega_{1}$ and $\omega_{2}$ be two pure GVBS states of the same spin chain with single-site Hilbert space $\mathscr{H}=\mathbb{C}^{d}$. Denote by $\Psi^{(M, N)}$ and $\Phi^{(M, N)}$ any pair of nonzero vectors in the range of the local density matrices of $\omega_{1}$ and $\omega_{2}$ respectively (i.e., vectors in the local supports of $\omega_{1}$ and $\omega_{2}$ on the interval $[M, N]$ as defined in (3.7)). Then, if $\omega_{1} \neq \omega_{2}$,

$$
\lim _{N-M \rightarrow \infty} \frac{\left\langle\Psi^{(M, N)} \mid A \Phi^{(M, N)}\right\rangle}{\left\|\Psi^{(M, N)}\right\|\left\|\Phi^{(M, N)}\right\|}=0
$$

for all local observables $A$.

We believe that this lemma can be proved using general disjointness and orthogonality properties of pure translation invariant states, without explicit reference to GVBS states. The "proof by computation" below has the advantage that it also shows how to compute the (in general non-vanishing) inner products of finite-volume support vectors. 
Proof. Before we start developing the argument, we collect the properties of pure GVBS states that we will need for this proof.

For $i=1,2$ let $\omega_{i}$ be given in terms of an isometry $V_{l}: \mathbb{C}^{k_{l}} \rightarrow \mathbb{C}^{d} \otimes \mathbb{C}^{k_{l}}$ (see (3.5) and a $k_{i} \times k_{i}$ density matrix $\rho_{i}$, and assume that these generating objects be minimal in the sense of [70] (see also Sect. 3). Then, it follows from [70] Theorem 1.5 that the maps defined by

$$
\mathbb{P}_{l}(B)=V_{l}^{*} \mathbb{1} \otimes B V_{l}, \quad B \in \mathscr{M}_{k_{l}}
$$

(where, as before, $\mathscr{M}_{k}$ denotes the complex $k \times k$ matrices) have trivial peripheral spectrum, i.e., 1 is their only eigenvalue with modulus $=1=\left\|\mathbb{P}_{i}\right\|$, and it is nondegenerate. The corresponding eigenvector is $\mathbb{1} \in \mathscr{M}_{k}$, i.e,

$$
\mathbb{P}_{i}(\mathbb{1})=\mathbb{1}
$$

The map $\mathbb{P}_{l}$ leaves the state $\rho_{l}$ invariant in the sense that

$$
\operatorname{Tr} \rho_{l} \mathbb{P}_{i}(B)=\operatorname{Tr} \rho_{i} B,
$$

and $\rho_{i}$ is the unique density matrix satisfying this equation. The $\rho_{i}$ are faithful states ([14], Lemma 2.5). In particular, $\rho_{i}$ is invertible.

The local support spaces of the state $\omega_{l}$ (i.e., the ranges of the local density matrices $\left.\rho_{[M, N]}\right)$ are spanned by the vectors of the form

$$
\Omega_{i}^{(n)}\left(\chi_{l}^{L}, \chi_{l}^{R}\right) \equiv \underbrace{W_{i} \otimes \cdots \otimes W_{i}}_{n+1}(\chi_{i}^{L} \otimes \underbrace{\varphi_{l} \otimes \cdots \otimes \varphi_{l}}_{n} \otimes \chi_{i}^{R}),
$$

where $n=N-M, \chi_{l}^{L}, \chi_{i}^{R} \in \mathbb{C}^{k_{l}}$ are arbitrary, $\varphi_{i} \in \mathbb{C}^{k_{i}} \times \mathbb{C}^{k_{l}}$ is defined in terms of $\rho_{l}$ (it is the GNS vector of the state $\rho_{l}$; see the proof of Proposition 2.7 in [14]). $W_{i}: \mathbb{C}^{k_{i}} \otimes \mathbb{C}^{k_{t}} \rightarrow \mathbb{C}^{d}$ is defined in terms of $\rho_{i}$ and $V_{i}$. The crucial relation is

$$
V_{l}^{*} A \otimes B V_{i}=\left(\operatorname{id}_{H_{k_{i}}} \otimes\left\langle\varphi_{l}|\cdot| \varphi_{l}\right\rangle\right)\left(W_{l}^{*} A W_{l}\right) \otimes B
$$

for all $A \in \mathscr{M}_{d}$ and $B \in \mathscr{M}_{k_{1}}$.

As the dimensions of the local support spaces are finite and independent of the size of the interval (as long as the interval is large enough), we can suffice with proving (4.4) for the spanning set of vectors of the form $\Psi^{(M, N)}=\Omega_{1}^{(N-M)}\left(\chi^{L}, \chi^{R}\right)$ and $\Phi^{(M, N)}=\Omega_{2}^{(N-M)}\left(\chi^{L}, \chi^{R}\right)$, as defined in (4.7).

For simplicity let us first consider the case $A=\mathbb{1}$ and put $n=N-M$. Then

$$
\begin{aligned}
\left\langle\Omega_{1}^{(n)}\left(\chi_{1}^{L}, \chi_{1}^{R}\right) \mid \Omega_{2}^{(n)}\left(\chi_{2}^{L}, \chi_{2}^{R}\right)\right\rangle= & \langle\chi_{1}^{L} \otimes \underbrace{\varphi_{1} \otimes \cdots \otimes \varphi_{1}}_{n} \otimes \chi_{1}^{R}|\underbrace{W_{1}^{*} W_{2} \otimes \cdots \otimes W_{1}^{*} W_{2}}_{n+1}| \\
& \times \chi_{2}^{L} \otimes \underbrace{\varphi_{2} \otimes \cdots \otimes \varphi_{2}}_{n} \otimes \chi_{2}^{R}\rangle .
\end{aligned}
$$

Define a linear transformation $\mathbb{P}_{12}$ of $\mathscr{M}_{k_{1}, k_{2}}$, the $k_{1} \times k_{2}$ matrices, by

$$
\mathbb{P}_{12}(B)=\left(\operatorname{id} \otimes\left\langle\varphi_{1}|\cdot| \varphi_{2}\right\rangle\right)\left(W_{1}^{*} W_{2} \otimes B\right)=V_{1}^{*} \mathbb{1} \otimes B V_{2}, \quad B \in \mathscr{M}_{k_{1}, k_{2}},
$$


where $V_{1}$ and $V_{2}$ are the isometries satisfying (4.8). The inner product (4.9) can then be written in the form

$$
\left\langle\chi_{1}^{L}\left|\mathbb{P}_{12}^{n}\left(\left(\mathrm{id} \otimes\left\langle\chi_{1}^{R}|\cdot| \chi_{2}^{R}\right\rangle\right)\left(W_{1}^{*} W_{2}\right)\right)\right| \chi_{2}^{L}\right\rangle .
$$

In general the mat:ix element between local support vectors of a local observable $A$ is of the following form:

$$
\left\langle\Omega_{1}^{(n)}\left(\chi_{1}^{L}, \chi_{1}^{R}\right) \mid A \Omega_{2}^{(n)}\left(\chi_{2}^{L}, \chi_{2}^{R}\right)\right\rangle=\operatorname{Tr} C^{*} \mathbb{P}_{12}^{n-m-1} \circ \mathbb{F}_{A}^{(l)} \circ \mathbb{P}_{12}^{m}(B)
$$

for some $C, B \in \mathscr{M}_{k_{1}, k_{2}}$, where $l$ is the length of the interval on which $A$ acts nontrivially, and $\mathbb{F}_{A}^{(l)}$ is the linear transformation of $\mathscr{M}_{k_{1}, k_{2}}$ defined by

$$
\mathbb{F}_{A}^{(l)}(B)=\mathbb{F}_{A_{l}} \circ \cdots \circ \mathbb{F}_{A_{l}}(B), \quad \text { for } A=A_{1} \otimes \cdots \otimes A_{l}, \quad A_{1}, \ldots, A_{l} \in \mathscr{M}_{d}
$$

and

$$
\mathbb{F}_{A}(B)=V_{1}^{*} A \otimes B V_{2}, \quad A \in \mathscr{U}_{d}, B \in \mathscr{M}_{k_{1}, h_{2}} .
$$

The norms of the vectors $\Omega_{l}^{(n)}\left(\chi^{L}, \chi^{R}\right)$ can be calculated in the same way:

$$
\left\|\Omega_{l}^{(n)}\left(\chi^{L}, \chi^{R}\right)\right\|^{2}=\operatorname{Tr}\left|\chi^{L}\right\rangle\left\langle\chi^{L}\right| \mathbb{P}_{l}^{n}\left(\left(\mathrm{id} \otimes\left\langle\chi^{R}|\cdot| \chi^{R}\right\rangle\right)\left(W_{l}^{*} W_{l}\right)\right)
$$

It is straightforward to show from this relation that there are constants $C_{1}, C_{2}>0$ such that

$$
C_{1}\left\|\chi^{L}\right\|\left\|\chi^{R}\right\| \leqq\left\|\Omega_{i}^{(n)}\left(\chi^{L}, \chi^{R}\right)\right\| \leqq C_{2}\left\|\chi^{L}\right\|\left\|\chi^{R}\right\|
$$

See [14] for the details. Because of the bounds (4.11) and the considerations above, the statement of the lemma will follow if we show that, if $\omega_{1} \neq \omega_{2}$,

$$
\lim _{n \rightarrow \infty}\left\|\mathbb{P}_{12}^{n}\right\|=0
$$

where $\|\cdot\|$ denotes the usual norm of linear transformations $\mathbb{P}$ of $\mathscr{M}_{k_{1} \cdot k_{2}}$ considered as a Banach space:

$$
\|\mathbb{P}\|=\sup _{0 \neq B \in \|_{k} k_{2}} \frac{\|\mathbb{P}(B)\|}{\|B\|} .
$$

We will make a convenient choice for the norm on $\mathscr{M}_{k_{1}, k_{2}}$ below.

When $\omega_{1}=\omega_{2}$ there is always a unitary $U: \mathbb{C}^{h_{2}} \rightarrow \mathbb{C}^{k_{1}}$ such that

$$
V_{2}=\left(\mathbb{1} \otimes U^{*}\right) V_{1} U \text {. }
$$

In particular $k_{1}=k_{2}$. This is part of Theorem 1.5 of [70]. From (4.13) it follows that, in the case $\omega_{1}=\omega_{2}, \mathbb{P}_{12}(U)=U$ and hence $\left\|\mathbb{P}_{12}^{n}\right\| \geqq 1$.

We now show that in general the spectral radius of $\mathbb{P}_{12}$ is $\leqq 1$. More specifically we show that $\left\|\mathbb{P}_{12}\right\| \leqq 1$ if we use the norm on $\|_{k_{1}, k_{2}}$ defined by the state $\rho_{2}$ :

$$
\|B\|=\sqrt{\operatorname{Tr} \rho_{2} B^{*} B}, \quad B \in \|_{h_{1}, h_{2}} .
$$

This follows from Schwarz's inequality and the properties of $\mathbb{P}_{1}$ and $\mathbb{P}_{2}$. For all $B, C \in \mathbb{H}_{h_{1}, k_{2},}$,

$$
\begin{aligned}
\left|\operatorname{Tr} C^{*} \mathbb{P}_{12}(B)\right|^{2} & \leqq \operatorname{Tr} \rho_{2} C^{*} V_{1}^{*} V_{1} C \operatorname{Tr} \rho_{2} V_{2}^{*}\left(\mathbb{1} \otimes B^{*} B\right) V_{2} \\
& =\operatorname{Tr} \rho_{2} C^{*} C \operatorname{Tr} \rho_{2} B^{*} B .
\end{aligned}
$$


The last equality is obtained by using (4.5) for the map $\mathbb{P}_{1}$ and (4.6) for the map $\mathbb{P}_{2}$. Putting $C=\mathbb{P}_{12}(B)$ in (4.14) yields

$$
\left\|\mathbb{P}_{12}(B)\right\|^{4} \leqq\left\|\mathbb{P}_{12}(B)\right\|^{2}\|B\|^{2},
$$

proving that indeed $\left\|\mathbb{P}_{12}\right\| \leqq 1$.

When the spectral radius of $\mathbb{P}_{12}$ is strictly less than 1 , we have that $\left\|\mathbb{P}_{12}^{m}\right\|^{1 / m}<1$ for some large enough power $m$. In this case (4.14) follows and the lemma is proved.

When $\mathbb{P}_{12}$ has spectral radius $=1$, we complete the proof by showing that one necessarily has $\omega_{1}=\omega_{2}$. In that case, $\mathbb{P}_{12}$ has an eigenvalue $\lambda$ with $|\lambda|=1$, i.e., there is a $0 \neq B \in \mathscr{M}_{k_{1}, k_{2}}$ such that

$$
\mathbb{P}_{12}(B)=V_{1}^{*} \mathbb{1}(\otimes B) V_{2}=\lambda B .
$$

This implies $\mathbb{P}_{2}\left(B^{*} B\right)=B^{*} B$ by the following argument:

$$
\operatorname{Tr} \rho_{2} V_{2}^{*}\left(\mathbb{1} \otimes B^{*} B\right) V_{2}=\operatorname{Tr} \rho_{2} B^{*} B=\operatorname{Tr} \rho_{2} \mathbb{P}_{12}(B)^{*} \mathbb{P}_{12}(B),
$$

where for the first equality we used (4.6) for $\mathbb{P}_{2}$, and the second equality follows from (4.15). Equation (4.16) can be written as

$$
\operatorname{Tr} \rho_{2} V_{2}^{*}\left(\mathbb{1} \otimes B^{*}\right)\left(\mathbb{1}-V_{1}^{*} V_{1}\right)(\mathbb{1} \otimes B) V_{2}=0 .
$$

This is the expectation of a positive operator in the faithful state $\rho_{2}$, and hence

$$
V_{2}^{*}\left(\mathbb{1} \otimes B^{*} B\right) V_{2}=\mathbb{P}_{12}(B)^{*} \mathbb{P}_{12}(B)=B^{*} B .
$$

The eigenvalue 1 of $\mathbb{P}_{2}$ is non-degenerate and therefore, by (4.5),

$$
B^{*} B=\mu \mathbb{1} \in \mathscr{M}_{k_{2}}
$$

for some $0 \neq \mu \in \mathbb{C}$. By interchanging the roles of $\omega_{1}$ and $\omega_{2}$ and observing that $\mathbb{P}_{21}\left(B^{*}\right)=\bar{\lambda} B^{*}$, the previous argument also shows that

$$
B B^{*}=\mu^{\prime} \mathbb{1} \in \mathscr{M}_{k_{1}} .
$$

Together (4.17) and (4.18) show that $\mu=\mu^{\prime}>0$ and that $U \equiv \mu^{-1 / 2} B$ is unitary. In particular it follows that $k_{1}=k_{2}$.

For the eigenvector $U$ (or $B$ for that matter) of $\mathbb{P}_{12}$ one has equality in the Schwarz's inequality (4.14) with $C=B=U$, and therefore

$$
V_{1} U \rho_{2}^{1 / 2}=\mu^{\prime \prime}(\mathbb{1} \otimes U) V_{2} \rho_{2}^{1 / 2}
$$

for some complex constant $\mu^{\prime \prime}$. As $\rho_{2}$ is invertible this implies that there is a unitary, which we again denote by $U$, which intertwines the isometries $V_{1}$ and $V_{2}$ in the following sense:

$$
V_{1} U=(\mathbb{1} \otimes U) V_{2} .
$$

It follows immediately that $\mathbb{P}_{1}$ and $\mathbb{P}_{2}$ are unitarily equivalent and, by uniqueness of the invariant state, also that $\rho_{2}=U^{*} \rho_{1} U$. It is then straightforward to check, using (3.4), that $\omega_{1}=\omega_{2}$.

Consider any state $\omega$ which is a convex combination of states $\omega_{1}, \ldots, \omega_{n}$, i.e., $\omega=\sum_{\alpha=1}^{n} t_{\alpha} \omega_{\alpha}$, with $t_{\alpha}>0$ for $\alpha=1, \ldots, n$. Then, the local support spaces $\mathscr{G}_{\Lambda}$ of $\omega$ will be given by: $\mathscr{G}_{\Lambda}=\bigvee_{\alpha=1}^{n} \mathscr{G}_{\Lambda}^{\alpha}$, where the $\mathscr{G}_{\Lambda}^{\alpha}$ are the support spaces of 
the $\omega_{\alpha}$. The following proposition shows that the spaces $\mathscr{G}_{\Lambda}$ inherit the intersection property from the spaces $\mathscr{G}_{\Lambda}^{\alpha}$. The only extra property of the $\mathscr{G}_{\Lambda}^{\alpha}$ needed to prove this is a certain estimate on the overlap between them. In particular the states are not assumed to be GVBS states (it is an open question whether the intersection property of its support spaces implies that a state is GVBS). The proof of the proposition follows from two lemmas: Lemma 8 which gives two equivalent formulations of the intersection property, and Lemma 9 which is an elementary inequality for the overlap of a span of subspaces in terms of the overlap of the subspaces.

7. Proposition. For $n \geqq 2$, let $\omega_{1}, \ldots, \omega_{n}$ be $n$ distinct translation invariant states of a spin chain, whose support spaces satisfy the intersection property (3.10) for some $m_{0}$, i.e., for all $l, m, r, l \leqq 1, m \geqq m_{0}$, and $r \geqq m$ :

$$
\left(\mathscr{G}_{[l, r]}^{\alpha}=\mathscr{G}_{[l, m]}^{\alpha} \otimes \mathscr{H}_{[m+1, r]}\right) \cap\left(\mathscr{H}_{[l, 0]} \otimes \mathscr{G}_{[1, r]}\right) .
$$

Furthermore assume that for $\alpha \neq \beta$,

$$
\mathcal{O}\left(\mathscr{G}_{\left[1, m_{0}\right]}^{\alpha}, \mathscr{G}_{\left[1, m_{0}\right]}^{\beta}\right)<\frac{1}{n-1} .
$$

Then the spaces $\mathscr{G}_{\Lambda}=\bigvee_{\alpha=1}^{n} \mathscr{G}_{\Lambda}^{\alpha}$ satisfy the same intersection property (4.19).

Proof. By Lemma 8 and property ii) of the overlap we only have to prove that for all $\alpha$,

$$
\mathcal{O}\left(\mathscr{K}^{\alpha}, \bigvee_{\beta \neq \alpha} \mathscr{K}^{\beta}\right)<1,
$$

where $\mathscr{K}^{\alpha}=\left(\mathscr{G}_{[l, m]}^{\alpha} \otimes \mathscr{H}_{[m+1, r]}\right) \vee\left(\mathscr{H}_{[l, 0]} \otimes \mathscr{G}_{[1, r]}^{\alpha}\right)$, for $\alpha=1, \ldots, n$. As $\mathscr{K}^{\alpha} \subset \mathscr{G}_{[1, m]}^{\alpha}$ $\subset \mathscr{G}_{\left[1, m_{0}\right]}^{\alpha}$, and due to property vi) of the overlap, it is sufficient to prove

$$
\mathcal{O}\left(\mathscr{G}_{\left[1, m_{0}\right]}^{\alpha}, \bigvee_{\beta \neq \alpha} \mathscr{G}_{\left[1, m_{0}\right]}^{\beta}\right)<1
$$

for all $\alpha=1, \ldots, n$. This follows from Lemma 9 and the assumption on the mutual overlaps of the $\mathscr{G}_{\left[1, m_{0}\right]}^{\alpha}$ stated in the proposition.

A family of subspaces $\left\{\mathscr{G}^{\alpha}\right\}$ is called independent if for any $\psi \in \bigvee_{\alpha} \mathscr{G}^{\alpha}$ of the form $\psi=\sum_{\alpha} \psi^{\alpha}$ with $\psi^{\alpha} \in \mathscr{G}^{\alpha}$, one has $\psi=0 \Rightarrow \psi^{\alpha}=0$ for all $\alpha$ or, equivalently, if the decomposition $\psi=\sum_{\alpha} \psi_{\alpha}$ is unique. The property of independence is also equivalent with

$$
\mathscr{G}^{\beta} \cap \bigvee_{\alpha \neq \beta} \mathscr{G}^{\alpha}=\{0\} \quad \text { for all } \beta .
$$

The following provides us with two equivalent formulations of the intersection property.

8. Lemma. Let $\mathscr{H}_{L}, \mathscr{H}_{M}$ and $\mathscr{H}_{R}$ be Hilbert spaces and $I$ an index set, and let $\left\{\mathscr{G}_{L M}^{\alpha} \subset \mathscr{H}_{L} \otimes \mathscr{H}_{M} \mid \alpha \in I\right\}$ and $\left\{\mathscr{G}_{M R}^{\alpha} \subset \mathscr{H}_{M} \otimes \mathscr{H}_{R} \mid \alpha \in I\right\}$ be two families of independent subspaces. Then the following three properties are equivalent:

i) $\left(\bigvee_{\alpha} \mathscr{G}_{L M}^{\alpha} \otimes \mathscr{H}_{R}\right) \cap\left(\mathscr{H}_{L} \otimes \bigvee_{\alpha} \mathscr{G}_{M R}^{\alpha}\right)=\bigvee_{\alpha}\left(\mathscr{G}_{L M}^{\alpha} \otimes \mathscr{H}_{R}\right) \cap\left(\mathscr{H}_{L} \otimes \mathscr{G}_{M R}^{\alpha}\right)$

ii) $\left(\left(\mathscr{G}^{\beta} \otimes \mathscr{H}_{R}\right) \vee\left(\mathscr{H}_{L} \otimes \mathscr{G}_{M R}^{\beta}\right)\right) \cap \bigvee_{\alpha \neq \beta}\left(\mathscr{G}_{L M}^{\alpha} \otimes \mathscr{H}_{R}\right) \vee\left(\mathscr{H}_{L} \otimes \mathscr{G}_{M R}^{\alpha}\right)=\{0\}$ for all $\beta \in I$. 
iii) The subspaces $\left(\mathscr{G}_{L M}^{\alpha} \otimes \mathscr{H}_{R}\right) \vee\left(\mathscr{H}_{L} \otimes \mathscr{G}_{M R}^{\alpha}\right)$ of $\mathscr{H}_{L} \otimes \mathscr{H}_{M} \otimes \mathscr{H}_{R}$ also form an independent family.

Proof.

i) $\Rightarrow$ ii) Define for all $\alpha$ the space $\mathscr{G}_{L M R}^{\alpha}$ by

$$
\mathscr{G}_{L M R}^{\alpha}=\left(\mathscr{G}_{L M}^{\alpha} \otimes \mathscr{H}_{R}\right) \cap\left(\mathscr{H}_{L} \otimes \mathscr{G}_{M R}^{\alpha}\right) .
$$

Take any $\beta \in I$ and any

$$
\psi \in\left(\mathscr{G}_{L M}^{\beta} \otimes \mathscr{H}_{R}\right) \vee\left(\mathscr{H}_{L} \otimes \mathscr{G}_{M R}^{\beta}\right) \cap \bigvee_{\alpha \neq \beta}\left(\mathscr{G}_{L M}^{\alpha} \otimes \mathscr{H}_{R}\right) \vee\left(\mathscr{H}_{L} \otimes \mathscr{G}_{M R}^{\alpha}\right)
$$

Then there exist $\psi_{L M}^{\alpha} \in \mathscr{G}_{L M}^{\alpha} \otimes \mathscr{H}_{R}$ and $\psi_{M R}^{\alpha} \in \mathscr{H}_{L} \otimes \mathscr{G}_{M R}^{\alpha}$, for all $\alpha \in I$, such that $\psi=\psi_{L M}^{\beta}+\psi_{M R}^{\beta}=\sum_{\alpha \neq \beta} \psi_{L M}^{\alpha}+\psi_{M R}^{\alpha}$. Put $\xi=-\psi_{L M}^{\beta}+\sum_{\alpha \neq \beta} \psi_{L M}^{\alpha}=\psi_{M R}^{\beta}-\sum_{\alpha \neq \beta} \psi_{M R}^{\alpha}$. Then obviously

$$
\xi \in \bigvee_{\alpha}\left(\mathscr{G}_{L M}^{\alpha} \otimes \mathscr{H}_{R}\right) \cap \bigvee_{\alpha}\left(\mathscr{H}_{L} \otimes \mathscr{G}_{M R}^{\alpha}\right),
$$

and hence, by i), $\xi \in \bigvee_{\alpha} \mathscr{G}_{L M R}^{\alpha}$. From the definition of the spaces $\mathscr{G}_{L M R}^{\alpha}$ and the independence of either the $\mathscr{G}_{L M}^{\alpha}$ or the $\mathscr{G}_{M R}^{\alpha}$ it follows that the spaces $\mathscr{G}_{L M R}^{\alpha}$ also form a family of independent subspaces of $\mathscr{H}_{L} \otimes \mathscr{H}_{M} \otimes \mathscr{H}_{R}$. Using this one immediately concludes that in the decompositions $\xi=\sum_{\alpha} \xi_{L M}^{\alpha}=\sum_{\alpha} \xi_{L M R}^{\alpha}=\sum_{\alpha} \xi_{M R}^{\alpha}$, with $\xi_{L M}^{\alpha} \in \mathscr{G}_{L M}^{\alpha} \otimes \mathscr{H}_{R}, \xi_{L M R}^{\alpha} \in \mathscr{G}_{L M R}^{\alpha}$ and $\xi_{M R}^{\alpha} \in \mathscr{H}_{L} \otimes \mathscr{G}_{M R}^{\alpha}$, one must actually have $\xi_{L M}^{\alpha}=\xi_{L M R}^{\alpha}=\xi_{M R}^{\alpha}$ for all $\alpha \in I$. Comparing the definition of $\xi$ with the employed decompositions of $\psi$ we obtain $\psi=\psi_{L M}^{\beta}+\psi_{M R}^{\beta}=-\xi_{L M}^{\beta}+\xi_{M R}^{\beta}=0$.

ii) $\Rightarrow$ i) Now take

$$
\psi \in \bigvee_{\alpha}\left(\mathscr{G}_{L M}^{\alpha} \otimes \mathscr{H}_{R}\right) \cap \bigvee_{\alpha}\left(\mathscr{H}_{L} \otimes \mathscr{G}_{M R}^{\alpha}\right) .
$$

$\psi$ then has the decompositions $\psi=\sum_{\alpha} \psi_{L M}^{\alpha}=\sum_{\alpha} \psi_{M R}^{\alpha}$, with $\psi_{L M}^{\alpha} \in \mathscr{G}_{L M}^{\alpha} \otimes \mathscr{H}_{R}$ and $\psi_{M R}^{\alpha} \in \mathscr{H}_{L} \otimes \mathscr{G}_{M R}^{\alpha}$ for all $\alpha \in I$. For any $\beta \in I$, put $\xi=\psi_{L M}^{\beta}-\psi_{M R}^{\beta}=\sum_{\alpha \neq \beta} \psi_{M R}^{\alpha}-$ $\psi_{L M}^{\alpha}$. It is then obvious that

$$
\xi \in\left(\mathscr{G}^{\beta} \otimes \mathscr{H}_{R}\right) \vee\left(\mathscr{H}_{L} \otimes \mathscr{G}_{M R}^{\beta}\right) \cap \bigvee_{\alpha \neq \beta}\left(\mathscr{G}^{\alpha} \otimes \mathscr{H}_{R}\right) \vee\left(\mathscr{H}_{L} \otimes \mathscr{G}_{M R}^{\alpha}\right),
$$

and by ii) this implies $\psi_{L M}^{\beta}=\psi_{M R}^{\beta}$. As $\beta \in I$ is arbitrary, we can conclude that $\psi \in \bigvee_{\alpha} \mathscr{G}_{L M R}^{\alpha}$. So, we have shown that

$$
\bigvee_{\alpha}\left(\mathscr{G}_{L M}^{\alpha} \otimes \mathscr{H}_{R}\right) \cap \bigvee_{\alpha}\left(\mathscr{H}_{L} \otimes \mathscr{G}_{M R}^{\alpha}\right) \subset \bigvee_{\alpha} \mathscr{G}_{L M R}^{\alpha}
$$

The opposite inclusion is trivial from the definition of the $\mathscr{G}_{L M R}^{\alpha}$. lemma.

ii) $\Leftrightarrow$ iii) This equivalence follows immediately from the remark preceding the

By property ii) of the overlap independence of a family of subspaces $\mathscr{G}^{\alpha}$ is equivalent with

$$
\mathcal{O}\left(\mathscr{G}^{\alpha}, \bigvee_{\beta \neq \alpha} \mathscr{G}^{\beta}\right)<1 \text { for all } \alpha \text {. }
$$


This inequality will hold when the mutual overlaps of the spaces $\mathscr{G}^{\alpha}$ are sufficiently small, as is shown in the next lemma.

9. Lemma. Let $\mathscr{G}^{1}, \ldots, \mathscr{G}^{n}$ be $n$ subspaces of a Hilbert space $\mathscr{H}$. Assume

$$
\mathcal{O}\left(\mathscr{G}^{\alpha}, \mathscr{G}^{\beta}\right) \leqq \varepsilon_{\alpha \beta} .
$$

Then, if $\|B\|<1$,

$$
\mathcal{O}\left(\mathscr{G}^{n}, \bigvee_{\alpha=1}^{n-1} \mathscr{G}^{\alpha}\right) \leqq \frac{\|a\|}{\sqrt{1-\|B\|}}
$$

where $a \in \mathbb{R}^{n-1}$ is the vector with components $a_{\alpha}=\varepsilon_{n x}, \alpha=1, \ldots, n-1$, and $B$ is the $(n-1) \times(n-1)$ matrix with entries

$$
B_{\alpha \beta}=\left(1-\delta_{\alpha \beta}\right) \varepsilon_{\alpha \beta} \quad \alpha, \beta=1, \ldots, n-1 .
$$

In particular if all $\varepsilon_{\alpha \beta} \leqq \varepsilon \leqq 1 /(n-1)$ we have

$$
\mathcal{O}\left(\mathscr{G}^{n}, \bigvee_{\alpha=1}^{n-1} \mathscr{G}^{\alpha}\right) \leqq \frac{\varepsilon \sqrt{n-1}}{\sqrt{1-\varepsilon(n-2)}} \leqq 1
$$

Proof. The proof is an elementary application of Schwarz's inequality. Let $\psi_{\alpha} \in$ $\mathscr{G}^{\alpha}, \alpha, 1, \ldots, n$ be such that

$$
\left\|\sum_{\alpha=1}^{n-1} \psi_{\alpha}\right\|=1 \quad \text { and } \quad\left\|\psi_{n}\right\|=1
$$

We then have to prove that

$$
\left|\left\langle\psi_{n} \mid \sum_{\alpha=1}^{n-1} \psi_{\alpha}\right\rangle\right| \leqq \frac{\|a\|}{\sqrt{1-\|B\|}}
$$

As $\left\|\psi_{n}\right\|=1$ we have

$$
\left|\left\langle\psi_{n} \mid \sum_{\alpha=1}^{n-1} \psi_{\alpha}\right\rangle\right| \leqq \sum_{\alpha=1}^{n-1} \varepsilon_{n \alpha}\left\|\psi_{\alpha}\right\| \leqq\|a\| \sqrt{\sum_{\alpha=1}^{n-1}\left\|\psi_{\alpha}\right\|^{2}} .
$$

Using the definition of the matrix $B$ we derive

$$
\left|\left\|\sum_{\alpha=1}^{n-1} \psi_{\alpha}\right\|^{2}-\sum_{\alpha=1}^{n-1}\left\|\psi_{\alpha}\right\|^{2}\right| \leqq \sum_{\alpha \neq \beta, 1}^{n-1}\left|\left\langle\psi_{\alpha} \mid \psi_{\beta}\right\rangle\right| \leqq \sum_{\alpha \neq \beta, 1}^{n-1} \varepsilon_{\alpha \beta}\left\|\psi_{\alpha}\right\|\left\|\psi_{\beta}\right\| \leqq\|B\| \sum_{\alpha=1}^{n-1}\left\|\psi_{\alpha}\right\|^{2}
$$

As $\left\|\sum_{\alpha=1}^{n-1} \psi_{\alpha}\right\|=1$ this implies

$$
\sum_{\alpha=1}^{n-1}\left\|\psi_{\alpha}\right\|^{2} \leqq \frac{1}{1-\|B\|}
$$

Combined with (4.22) this proves (4.20).

It is obvious that if all $\varepsilon_{\alpha \beta} \leqq \varepsilon$, then $\|a\| \leqq \sqrt{n-1}$, and because $B$ is symmetric and has non-negative matrix elements, we also have

$$
\|B\| \leqq \varepsilon\left\|\left(1-\delta_{\alpha \beta}\right)_{\alpha, \beta=1}^{n-1}\right\|=\varepsilon(n-2) .
$$




\section{Existence of GVBS Interactions: The Proof of Theorem 1}

Recall that for a state $\omega$ of a quantum spin chain, $\mathscr{F}_{\omega}$ denotes the smallest $\left(w^{*}\right.$ closed) set of states of the chain that contains $\omega$ and that satisfies: for any three states $\eta, \eta_{1}$ and $\eta_{2}$ of the chain such that $\eta=t \eta_{1}+(1-t) \eta_{2}$ for some $t \in(0,1)$, one has $\eta \in \mathscr{F}_{\omega} \Leftrightarrow \eta_{1}$ and $\eta_{2} \in \mathscr{F}_{\omega}$. Let $0 \leqq h$ be an interaction of range $l$ and denote by $h_{i}, i \in \mathbb{Z}$, a copy of $h$ acting on the sites $i, i+1, \ldots, i+l-1$ of the chain. As before, we denote by $\mathscr{F}_{h}$ the set of states $\eta$ of the chain such that $\eta\left(h_{l}\right)=0$, for all $i \in \mathbb{Z}$. We also use the notation $\rho_{\Lambda}$ for the local density matrices of $\omega$ and the spaces $\mathscr{G}_{\Lambda}$ as defined in Sect. 3.

Let $h_{i}$ denote the translation over $i$ of a finite range interaction $h \geqq 0$. From the simple observation that

$$
\operatorname{ker}\left(H_{[M, N]} \equiv \sum_{i=M}^{N-l+1} h_{i}\right)=\bigcap_{i=M}^{N-l+1} \mathscr{H}_{[M, i-1]} \otimes \operatorname{ker} h \otimes \mathscr{H}_{[i+l, N]},
$$

it follows that the spaces $\mathscr{G}_{A}$ have the intersection property (3.10) iff there exists a finite range interaction $h \geqq 0$ such that $\mathscr{G}_{\Lambda}=\operatorname{ker} H_{\Lambda}$ for all finite intervals $\Lambda \subset \mathbb{Z}$. For the infinite volume states we have the following lemma.

10. Lemma. Let $\omega$ be a translation invariant state of a chain such that the local support spaces $\mathscr{G}_{\Lambda}$ of $\omega$ have the intersection property (3.10) for a certain $m_{0}$. Assume in addition that there exists a constant $\delta>0$ such that for all $M \leqq N \in$ $\mathbb{Z}, \rho_{[M, N]} \geqq \delta$ on its support, i.e.

$$
\left(\rho_{[M, N]}\right)^{2} \geqq \delta \rho_{[M, N]}
$$

Then there exists a finite range interaction $h \in\left(\mathscr{M}_{d}\right)^{\otimes m_{0}}$ such that $\mathscr{F}_{h}=\mathscr{F}_{\omega}$.

Proof. Let $l_{0}$ be an integer such that (3.10) holds. Define $h$ as the orthogonal projection onto $\mathscr{G}_{\left[1, l_{0}\right]}^{\perp}$. Then $h \geqq 0$ and $\omega\left(h_{l}\right)=0$ for all $i \in \mathbb{Z}$. For any $\eta \in \mathscr{F}_{\omega}$ there exists a $t \in(0,1)$ such that we can find a state $\eta^{\prime}$ such that $\omega=t \eta+(1-t) \eta^{\prime}$. It follows that $\eta\left(h_{l}\right)=0$ for all $\eta \in \mathscr{F}_{\omega}$. Hence $\mathscr{F}_{\omega} \subset \mathscr{F}_{h}$.

In order to prove the opposite inclusion, take $\eta \in \mathscr{F}_{h}$. Then, by the intersection property, we must have that for any finite volume $\Lambda$ the restriction $\eta_{\Lambda}$ of $\eta$ is a substate of $\omega_{\Lambda}$, i.e. there exists a constant $C_{\Lambda}(\eta)>0$ such that

$$
\eta_{\Lambda} \leqq C_{\Lambda}(\eta) \omega_{\Lambda}
$$

The condition (5.1) implies that $C_{\Lambda}(\eta)$ can always be taken to $\delta^{-1}$, i.e. independent of $\Lambda$ and $\eta$. It follows that there exists a $t_{\Lambda} \in[\delta, 1]$, and a state $\eta_{\Lambda}^{\prime}$ such that

$$
\omega_{\Lambda}=t_{\Lambda} \eta_{\Lambda}+\left(1-t_{\Lambda}\right) \eta_{\Lambda}^{\prime} \text {. }
$$

Choose a sequence of intervals $\Lambda_{l}$, increasing to $\mathbb{Z}$, such that $\lim _{i \rightarrow \infty} t_{\Lambda_{l}}$ exists and equals say $t$. Then $\omega=t \eta+(1-t) \eta^{\prime}$, where $\eta^{\prime}=\lim _{i} \eta_{\Lambda^{\prime}}^{\prime}$ is well-defined because of (5.3). As $t \geqq \delta>0$ we can conclude that $\eta \in \mathscr{F}_{\omega}$.

We now can complete the proof of Theorem 1 . 
Proof of Theorem 1. It follows from the decomposition theory of GVBS states that any GVBS state can be decomposed into a finite number of ergodic components, which are again GVBS states. So, if $\omega$ is a GVBS state, there are ergodic GVBS states $\omega_{1}, \ldots, \omega_{k}$ and convex combination coefficients $t_{1}, \ldots, t_{k}$, such that $\omega=\sum_{l=1}^{k} t_{l} \omega_{i}$. Furthermore any of these ergodic GVBS states $\omega_{i}$ has a decomposition into $p_{l}$ periodic states $\omega_{i, q}, q=1, \ldots, p_{i}$, with equal weights: $\omega_{i}=$ $p_{l}^{-1} \sum_{q=1}^{p_{l}} \omega_{l, q}$. The states $\omega_{l, q}$ are invariant under translation over $p_{i}$ lattice spacings: $\omega_{l, q} \circ \tau_{p_{l}}=\omega_{l, q}$, and one also has that $\omega_{l, q} \circ \tau_{1}=\omega_{i, q+1\left(\bmod p_{l}\right)}$. All the $\omega_{i, q}$ are pure GVBS states. For a proof of these properties see [14], or for a more complete account see [70] .

Let $p$ be the least common multiple of $p_{1}, \ldots, p_{k}$. Then all states $\omega_{l, q}$ are $\tau_{p^{-}}$ invariant. So, consider a regrouped chain where the sites correspond to intervals of length $p$ of the original chain. Now we are in a situation where Theorem 5 applies with $n=\sum_{l=1}^{k} p_{l}$. So, at the level of the regrouped chain we have the intersection property for the ground state spaces $\mathscr{G}_{\Lambda}$ of $\omega$. By Lemma 9 this implies that there exists the finite range interaction $h^{(p)} \in\left(\mathscr{M}_{d}^{\otimes p}\right)^{\otimes m_{0}}$, for some constant $m_{0}$, such that $\mathscr{F}_{\omega}=\left\{\eta \mid \eta\left(h_{p l}^{(p)}\right)=0\right.$ for all $\left.i \in \mathbb{Z}\right\}$. The condition (5.1) in Lemma 10 is satisfied for GVBS states because of (3.6). Here $h_{p i}^{(p)}$ acts on the sites $p i, p i+1, \ldots, p i+$ $p m_{0}+1$ of the original chain. This does not immediately yield a translation invariant Hamiltonian for which the states $\omega_{l, q}$ are the ground states. But the interaction can be made translation invariant by defining

$$
h_{l}=\sum_{q=0}^{p-1} h_{i+q}^{(p)} \in \mathscr{M}_{d}^{\otimes p m_{0}+p-1} .
$$

Observe that due to the translation invariance of $\omega$ we have:

$$
\omega\left(h_{l}\right)=\sum_{q=0}^{p-1}\left(\omega \circ \tau_{q}\right)\left(h_{i}^{(p)}\right)=0 .
$$

Hence also $\omega_{i, q}(h)=0$ for all $i=1, \ldots, k$ and $q=1, \ldots, p_{i}$. So we conclude that $\mathscr{F}_{\omega} \subset \mathscr{F}_{h}$. As $h \geqq h^{(p)}$ we certainly have $\mathscr{F}_{h} \subset\left\{\eta \mid \eta\left(h_{p i}^{(p)}\right)=0\right\}=\mathscr{F}_{\omega}$, hence $\mathscr{\mathscr { F }}_{h}=\mathscr{F}_{()}$.

From the arguments in the proof it is also clear that the interaction $h$ can be chosen such that the Hamiltonian is invariant under all symmetries of the set of states $\left\{\omega_{i} \mid i=1, \ldots, k\right\}$. This holds equally well for broken as for unbroken symmetries. By a broken symmetry we mean a symmetry transformation that does not leave invariant at least one of the pure components of the states $\omega_{i}$, but such that states in the set $\mathscr{F}_{\omega}$ are transformed into states of $\mathscr{F}_{\omega}$. An interesting consequence of this observation is that one can construct GVBS models with any kind of prescribed discrete symmetry, and such that this symmetry is spontaneously broken in the ground states of the model. The only requirement is that there exist translation invariant (or periodic) ergodic states of the chain (not necessarily GVBS states) which break the symmetry, and which belong to a finite orbit of the symmetry group, i.e., by application of the symmetry transformations one generates a finite number of different states. The only requirement is that the symmetries preserve the GVBS nature of the states, i.e., they should transform GVBS states into GVBS states. This is known to be the case for lattice translations, lattice reflections, and 
local symmetries described by unitary or anti-unitary transformations, which includes the following examples:

1) symmetries described by a finite group of unitaries $U \in \mathscr{M}_{d}$, acting on the observables as $A \mapsto\left(U^{*}\right)^{\otimes N} A U^{\otimes N}$, for $A$ an observable that lives on an interval of length $N$,

2) the translation symmetry of the chain,

3 ) the reflection symmetry $R$ of the chain given by $\mathbb{Z} \ni i \mapsto-i$ and its natural lifting to the algebra of observables. A necessary and sufficient condition for a pure GVBS state $\omega$ to be $R$-invariant is the following. Let $\omega$ be defined in terms of an isometry $V: \mathbb{C}^{k} \rightarrow \mathbb{C}^{d} \otimes \mathbb{C}^{k}$ by the formulae:

$$
\mathbb{E}_{A}(B)=V^{*} A \otimes B V, \text { for all } A \in \mathscr{M}_{d}, B \in \mathscr{M}_{k},
$$

and for $A_{1}, \ldots, A_{i} \in \mathscr{M}_{d}$

$$
\omega\left(A_{1} \otimes \cdots \otimes A_{n}\right)=\rho\left(\mathbb{E}_{A_{1}} \circ \cdots \circ \mathbb{E}_{A_{n}}\left(\mathbb{1}_{k}\right)\right),
$$

where $\rho$ is a state of $\mathscr{M}_{k}$ satisfying $\rho\left(\mathbb{E}_{\mathbb{l}}(B)\right)=\rho(B)$, and where $k$ is the smallest integer for which this is possible (see the minimality condition mentioned in Sect. 2 ). Then $\omega$ is $R$-invariant iff there exist orthonormal bases $\left\{f_{a}\right\}$ and $\left\{e_{i}\right\}$ of $\mathbb{C}^{d}$ and $\mathbb{C}^{k}$ respectively, and a unitary $U \in \mathscr{M}_{k}$, such that for all $a=1, \ldots, d, i, j=1, \ldots, k$, one has:

$$
\left\langle f_{a} \otimes U e_{l} \mid V U e_{j}\right\rangle=\left\langle f_{a} \otimes e_{j} \mid V e_{i}\right\rangle,
$$

If one fixes the vector $f_{a}$, the action of $V$ is given by a $k \times k$ matrix. The above relation says that there exists a single unitary $U$ which transforms these $d$ matrices of dimensions $k \times k$ into their transposes. This characterization is an application of the results in [70].

4) symmetries described by anti-unitaries as the charge conjugation and the chiral symmetry (cfr. Sect. 7 for an example).

Moreover any of the above symmetries can be considered after regrouping the chain first and, of course, one can also form products of the elementary symmetries described in 1-4).

\section{Existence of the Spectral Gap: The Proof of Theorem 2}

In order to prove the existence of a spectral gap for the GVBS models obtained in the previous section, we need to develop the arguments in the proof of Proposition 5 a little bit further. This is accomplished in Lemma 11 which, together with the results of [14], proves that condition $\mathrm{C}^{\prime}$ of Sect. 2 is satisfied. Conditions $\mathrm{C} 1$ and $\mathrm{C} 2$ are trivially satisfied in the situation at hand. Theorem 2 is then a direct consequence of Theorem 3, the properties of pure GVBS states proved in [14], and a simple argument to pass to the thermodynamic limit.

For convenience we define

$$
A_{m}^{\alpha}=\sup _{l \leqq 1, r \geqq m}\left\|G_{[l, r]}^{\alpha}-\left(G_{[l, m]}^{\alpha} \otimes \mathbb{1}_{[m+1, r]}\right)\left(\mathbb{1}_{[l, 0]} \otimes G_{[1, r]}^{\alpha}\right)\right\| .
$$

$A_{m}^{\alpha}$ is the best constant in the commutation property (3.15) for the ground state projections of pure state $\omega_{\alpha}$ on arbitrary finite intervals that overlap on $m$ sites. 
Combination of the results in [14] and in [70] proves that the $A_{m}^{\alpha}$ satisfy a bound of the form

$$
A_{m}^{\gamma} \leqq c \lambda^{m} \frac{1+c \lambda^{m}}{1-c \lambda^{m}}
$$

for some constants $C>0$ and $0<\lambda<1$ which depend on the state $\omega_{\alpha}$. $C$ can be taken to be equal to $k^{2}$, where $k$ is the dimension of the auxiliary space used to define the pure GVBS state $\omega_{\alpha}$ (see (3.1)). $\lambda$ can be any number satisfying $\lambda_{l}<\lambda<1$ for all eigenvalues $\lambda_{l} \neq 1$ of the transfer operator $\mathbb{P}$ for the state $\omega_{\alpha}$.

We now first derive the commutation property for the GVBS state $\omega$ that we need for the proof of Theorem 2 . Define for all $m \geqq 1$ the operator $X_{m}$ by

$$
X_{m}=\sum_{\alpha=1}^{n} G_{[1, m]}^{\alpha}
$$

For all $m$ such that

$$
\varepsilon_{\alpha \beta}(m) \equiv \mathcal{O}\left(\mathscr{G}_{[1, m]}^{\alpha}, \mathscr{G}_{[1, m]}^{\beta}\right)<\frac{1}{n-1},
$$

we define

$$
\delta_{m}=\frac{\|\varepsilon\|}{1-\|\varepsilon\|}
$$

where $\varepsilon=\left(\varepsilon_{x \beta}\right)$ is the $n \times n$ matrix of the mutual overlaps of the spaces $\mathscr{G}_{[1, m]}^{\alpha}$ and with 0 's on the diagonal, i.e., we put $\varepsilon_{\gamma \alpha}=0$ by definition. Note that if $\varepsilon_{\alpha \beta} \leqq \varepsilon$ for all $\alpha$, one has the simple bound $\|\varepsilon\| \leqq(n-1) \varepsilon$, and $\delta_{m} \leqq(n-1) \varepsilon /(1-(n-1) \varepsilon)$. From the properties of the overlap it immediately follows that the $\varepsilon_{\alpha \beta}$, and hence also $\delta_{m}$, are decreasing functions of $m$.

As before, $G_{\Lambda}$ denotes the orthogonal projection onto the space $\bigvee_{\alpha=1}^{n} \mathscr{G}_{\Lambda}^{\alpha}$.

11. Lemma. Let $m_{0}$ be such that

$$
\mathcal{O}\left(\mathscr{G}_{[1, m]}^{\alpha}, \mathscr{G}_{[1, m]}^{\beta}\right)<\frac{1}{2(n-1)} \text { for all } \alpha \neq \beta
$$

and let $\delta_{m}$ be defined as in (6.4) and let $X_{m}$ be the operators defined in (6.2). Then

i) for all $m \geqq m_{0}$ we have the bound

$$
\left\|X_{m}-G_{[1, m]}\right\| \leqq \frac{\delta_{m}}{1-\delta_{m}}
$$

ii) For all $m \geqq m_{0}, l \leqq 1, r \geqq m$ one has

$$
\left\|G_{[l, r]}-\left(G_{[l, m]} \otimes \mathbb{1}_{[m+1, r]}\right)\left(\mathbb{1}_{[l, 0]} \otimes G_{[1, r]}\right)\right\| \leqq 4 \frac{\delta_{m}}{\left(1-\delta_{m}\right)^{2}}+\sum_{\alpha=1}^{n} A_{m}^{\alpha}
$$


Proof. We will use the following bound for the proof of both i) and ii). Let $\varphi$ and $\psi$ be two vectors that are of the form $\varphi=\sum_{\alpha=1}^{n} \varphi_{\alpha}$ and $\psi=\sum_{\alpha=1}^{n} \psi_{\alpha}$, with $\varphi_{\alpha} \in \mathscr{G}_{\Lambda_{1}}^{\alpha}$ and $\psi_{\alpha} \in \mathscr{G}_{\Lambda_{2}}^{\alpha}$, where $\Lambda_{1}$ and $\Lambda_{2}$ are two finite volumes containing the interval $[1, m]$. We will show that

$$
\sum_{\alpha \neq \beta}\left|\left\langle\varphi_{\alpha} \mid \psi_{\beta}\right\rangle\right| \leqq \delta_{m}\|\varphi\|\|\psi\|
$$

As in the proof of Lemma 9, we use Schwarz's inequality to obtain

$$
\sum_{\alpha \neq \beta}\left|\left\langle\varphi_{\alpha} \mid \psi_{\beta}\right\rangle\right| \leqq \sum_{\alpha \neq \beta} \varepsilon_{\alpha \beta}\left\|\varphi_{\alpha}\right\|\left\|\psi_{\beta}\right\| \leqq\|\varepsilon\| \sqrt{\sum_{\alpha=1}^{n}\left\|\varphi_{\alpha}\right\|^{2} \sum_{\alpha=1}^{n}\left\|\psi_{\alpha}\right\|^{2}} .
$$

Applying this inequality for $\varphi_{\alpha}=\psi_{\alpha}$ one obtains

$$
\left|\left\|\sum_{\alpha=1}^{n} \varphi_{\alpha}\right\|^{2}-\sum_{\alpha=1}^{n}\left\|\varphi_{\alpha}\right\|^{2}\right| \leqq\|\varepsilon\| \sum_{\alpha=1}^{n}\left\|\varphi_{\alpha}\right\|^{2}
$$

and hence

$$
\sum_{x=1}^{n}\left\|\varphi_{x}\right\|^{2} \leqq \frac{1}{1-\|\varepsilon\|}\|\varphi\|^{2} .
$$

Combining (6.8) and (6.9) we obtain (6.7).

Proof of $i$. It is obvious that $X_{m}\left(\mathbb{1}_{[1, m]}-G_{[1, m]}\right)=0$, and therefore

$$
\left\|X_{m}-G_{[1, m]}\right\|=\sup _{\substack{\|\varphi\|=1 \\ G_{[1, m]} \varphi=\varphi}}\left\|\left(X_{m}-G_{[1, m]}\right) \varphi\right\| .
$$

Then, for $G_{[1, m]} \varphi=\varphi$, we can write $\varphi=\sum_{\alpha} \varphi_{\alpha}$, where $\varphi_{\alpha} \in \mathscr{G}_{[1, m]}^{\alpha}$, for $\alpha=$ $1, \ldots, n$ and we have $\left\|G_{[1, m]}^{\beta} \varphi_{\alpha}\right\| \leqq \varepsilon_{m}\left\|\varphi_{\alpha}\right\|$ if $\alpha \neq \beta$. So, we can estimate $\|\left(X_{m}-\right.$ $\left.G_{[1, m]}\right) \varphi\|=\| X_{m} \varphi-\varphi \|$ by estimating a quantity of the form (6.7):

$$
\begin{aligned}
\left\|X_{m} \varphi-\varphi\right\| & =\left\|\sum_{\alpha} \varphi_{\alpha}-\varphi+\sum_{\alpha \neq \beta} G_{[1, m]}^{\beta} \varphi_{\alpha}\right\|=\left\|\sum_{\alpha \neq \beta} G_{[1, m]}^{\beta} \varphi_{\alpha}\right\| \\
& =\sup _{\|\psi\|=1}\left|\sum_{\alpha \neq \beta}\left\langle\psi \mid G_{[1, m]}^{\beta} \varphi_{\alpha}\right\rangle\right| \leqq \sup _{\|\psi\|=1} \sum_{\alpha \neq \beta} \mid\left\langle\psi_{\beta} \mid \varphi_{\alpha}\right\rangle,
\end{aligned}
$$

where $\psi_{\beta}=G_{[1, m]}^{\beta} \psi$. By (6.7) this implies

$$
\left\|X_{m}-G_{[1, m]}\right\| \leqq \delta_{m}\left\|X_{m}\right\| .
$$

As $\left\|G_{[1, m]}\right\|=1$, this implies i).

Proof of $i$ ). By the triangle inequality it is sufficient to estimate the following sum of three terms:

$$
\begin{aligned}
& \left\|G_{[l, r]}-X_{r-l+1}\right\|+\left\|X_{r-l+1}-\sum_{\alpha}\left(G_{[l, m]}^{\alpha} \otimes \mathbb{1}_{[m+1, r]}\right)\left(\mathbb{1}_{[l, 0]} \otimes G_{[1, r]}^{\alpha}\right)\right\| \\
& \quad+\left\|\sum_{\alpha}\left(G_{[l, m]}^{\alpha} \otimes \mathbb{1}_{[m+1, r]}\right)\left(\mathbb{1}_{[l, 0]} \otimes G_{[1, r]}^{\alpha}\right)-\left(G_{[l, m]} \otimes \mathbb{1}_{[m+1, r]}\right)\left(\mathbb{1}_{[l, 0]} \otimes G_{[1, r]}\right)\right\| .
\end{aligned}
$$


The first term is bounded above by $\left\|G_{[l, r]}-X_{r-l+1}\right\|$ which we can estimate using i). The second term is estimated by $\sum_{\alpha=1}^{n} A_{m}^{\alpha}$. The third term can be treated as follows:

$$
\begin{aligned}
& \left\|\sum_{\alpha}\left(G_{[l, m]}^{\alpha} \otimes \mathbb{1}_{[m+1, r]}\right)\left(\mathbb{1}_{[l, 0]} \otimes G_{[1, r]}^{\alpha}\right)-\left(G_{[l, m]} \otimes \mathbb{1}_{[m+1, r]}\right)\left(\mathbb{1}_{[l, 0]} \otimes G_{[1, r]}\right)\right\| \\
& \leqq\left\|\left(G_{[l, m]} \otimes \mathbb{1}_{[m+1, r]}\right)\left(\mathbb{1}_{[l, 0]} \otimes G_{[1, r]}\right)-\left(X_{m-l+1} \otimes \mathbb{1}_{[m-1, r]}\right)\left(\mathbb{1}_{[l, 0]} \otimes X_{r}\right)\right\| \\
& +\left\|\sum_{\alpha \neq \beta}\left(G_{[l, m]}^{\alpha} \otimes \mathbb{1}_{[m+1, r]}\right)\left(\mathbb{1}_{[l, 0]} \otimes G_{[1, r]}^{\beta}\right)\right\| .
\end{aligned}
$$

By adding and subtracting $\left(G_{[l, m]} \otimes \mathbb{1}_{[1, r]}\right)\left(\mathbb{1}_{[l, 0]} \otimes X_{r}\right)$, the triangle inequality, and repeated use of $i)$ we find that the first term in the right side of the inequality above is bounded by

$$
\frac{\delta_{r-l+1}}{1-\delta_{r-l+1}}+\frac{\delta_{r-l+1}}{\left(1-\delta_{r-l+1}\right)^{2}}
$$

From (6.7) it follows that the second term is bounded by $\delta_{m} /\left(1-\delta_{m}\right)^{2}$. Collecting these estimates we obtain

$$
\begin{aligned}
\left\|G_{[l, r]}-\left(G_{[l, m]} \otimes \mathbb{1}_{[m+1, r]}\right)\left(\mathbb{1}_{[l, 0]} \otimes G_{[1, r]}\right)\right\| \leqq & \frac{2 \delta_{r-l+1}}{1-\delta_{r-l+1}}+\frac{\delta_{r-l+1}}{\left(1-\delta_{r-l+1}\right)^{2}} \\
& +\frac{\delta_{m}}{\left(1-\delta_{m}\right)^{2}}+\sum_{\alpha} A_{m}^{\alpha} .
\end{aligned}
$$

Because of the monotonicity of $\delta_{m}$ this implies ii).

We now prove the existence of a non-vanishing uniform lower bound for the spectral gap of the finite volume Hamiltonians $H_{[M, N]}$ defined by $H_{[M, N]}=$ $\sum_{l=M}^{N-l_{0}+1} h_{i}$, where $h$ is an interaction of range $l_{0}$ with the properties stated in Theorem 1. In fact the proof of this proposition does not rely anymore on the explicit GVBS structure of the ground states. The gap property is a direct consequence of the intersection property of the local support spaces $\mathscr{G}_{\Lambda}$ and the commutation property of the projections $G_{\Lambda}$ obtained in Lemma 11 . It is an interesting open question whether or not these properties by themselves imply that the state is a GVBS state.

12. Proposition (Proof of Theorem 2). Under the assumptions of Theorem 2 there exists a constant $\gamma>0$ such that for $N-M$ large enough, the gap between the lowest and the second lowest eigenvalue of $H_{[M, N]}$ exceeds $\gamma . \gamma$ is also a lower bound for the gap of the infinite system in any of its pure ground states. For $m$ large enough one has the following non-trivial lower bounds for $\gamma$ :

$$
\gamma \geqq \frac{\gamma_{2 m}}{2}\left(1-\eta_{m} \sqrt{2}\right)^{2}
$$

where $\gamma_{2 m}$ is the gap of the finite-volume Hamiltonian $H_{[1,2 m]}$ and $\eta_{m}$ satisfies

$$
\eta_{m} \leqq \frac{4(n-1) \varepsilon_{m}}{1-2(n-1) \varepsilon_{m}}+\sum_{\alpha=1}^{n} A_{m}^{\alpha},
$$

where $\varepsilon_{m}=\max _{\alpha \neq \beta} \mathcal{O}\left(\mathscr{G}_{[1, m]}^{\alpha}, \mathscr{G}_{[1, m]}^{\beta}\right)$, and the $A_{m}^{\alpha}$ satisfy the bound (6.1). 
Proof. From the previous results it is straightforward to check that the conditions $\mathrm{C} 1-\mathrm{C} 3^{\prime}$ (see Sect. 2) are satisfied with the constants $d=2$ and $\gamma_{2 m}$ as stated in the proposition. The uniform lower bound for the gap of the finite-volume Hamiltonians then follows directly from Theorem 3 .

In order to complete the proof we still have to show that the finite-volume estimate also applies to the gap of the GNS Hamiltonian of the infinite system in one of the ground states. This implication is rather trivial in the case at hand because any of the pure infinite volume ground states can be obtained as a limit of pure finite volume ground states of the Hamiltonians $H_{\Lambda}$ of which we proved that they have a uniformly bounded gap. Indeed, as the range of the interaction is finite, we have for any strictly local observable $X$ that is supported on the volume $\Lambda_{0}$,

$$
\begin{aligned}
\frac{\lim _{\Lambda \nearrow \mathbb{Z} \omega_{\alpha}\left(X^{*}\left[H_{\Lambda}, X\right]\right)}}{\omega_{\alpha}\left(X^{*} X\right)-\left|\omega_{\alpha}(X)\right|^{2}} & =\frac{\omega_{\alpha}\left(X^{*}\left[H_{\Lambda_{0}}, X\right]\right)}{\omega_{\alpha}\left(X^{*} X\right)-\left|\omega_{\alpha}(X)\right|^{2}} \\
& =\frac{\lim _{\Lambda_{1} \nearrow \mathbb{Z}} \omega_{\alpha, \Lambda_{1}}\left(X^{*}\left[H_{\Lambda_{1}}, X\right]\right)}{\lim _{\Lambda_{1}} \nearrow \mathbb{Z}\left(\omega_{\alpha, \Lambda_{1}}\left(X^{*} X\right)-\left|\omega_{\alpha, \Lambda_{1}}(X)\right|^{2}\right)} \geqq \gamma .
\end{aligned}
$$

Here the states $\omega_{\alpha, \Lambda_{1}}$ are of the form $\left\langle\psi_{\alpha, \Lambda_{1}}|\cdot| \psi_{\alpha, \Lambda_{1}}\right\rangle$ for some zero eigenvector $\psi_{\alpha, \Lambda_{1}}$ of $H_{\Lambda_{1}}$.

How fast the first term in (6.11) vanishes as $m \rightarrow \infty$ depends on how different the states $\omega_{\alpha}$ are on intervals of $m$ sites. The $A_{m}^{\alpha}$ also tend to 0 as $m \rightarrow \infty$, and has the same exponential behaviour as the truncated two-point correlation functions of the states $\omega_{\alpha}$. One should indeed expect two contributions of this kind to the commutation estimate ii) of Lemma 11. The support projections $G_{\Lambda}$ of $\omega$ cannot be expected to have better commutation properties than the projections of the components $\omega_{\alpha}$. On the other hand, even if the $\omega_{\alpha}$ have perfect commutation properties, the convex combination $\omega$ could fail to have these properties when the $\omega_{\alpha}$ are too close to one another. This happens, e.g., when there is breaking of a continuous symmetry as in the Heisenberg ferromagnet.

In the case $n=1$, i.e., GVBS models with a unique ground state, the first term in the right side of (6.11) vanishes for all $m$. The estimate on the infinite volume gap implied by (6.10) and (6.11), is then a little bit better than the one previously obtained in [14], which is with $\left(1-\sqrt{2} \eta_{m}\right)^{2}$ replaced by $\left(1-2 \eta_{m}\right)$. On the other hand, our estimate (6.10) suffers from the same overall factor $1 / 2$ that was also present in [14]. One therefore should expect the bounds to underestimate the infinite-volume gap by a factor $1 / 2$ at best. Note, however, that the factor $1 / 2$ is not present when Theorem $3 \mathrm{i})$ can be applied with $l<r$, which is the case, e.g., for the Heisenberg XXX (and also XXZ) ferromagnetic chain discussed in Lemma 4.

\section{Examples, Counterexamples, and Open Problems}

In this section we want to show how the general results of this paper, in particular Theorem 2, can be applied to a great variety of 1-D spin Hamiltonians. Although checking the conditions of Theorem 2 seems very simple, there is a subtle point that easily could be overlooked. Suppose one has a model defined in terms of a finite-range interaction $h \geqq 0$ and such that the infinite-volume zero-energy ground states of the model are all convex combinations of a finite number of GVBS-states. 
The subtlety is that this does not imply that $\operatorname{ker} H_{[M, N]}=\mathscr{G}_{[M, N]}$. In other words, the finite-volume Hamiltonians might have ground states that are not found back in the local support spaces of the infinite volume ground states. The thermodynamic limits of these additional ground do not go beyond the GVBS ground states we already had, but for a finite volume they are different. An example of this situation is given in Example 2. There we show that not only does Theorem 2 not apply, but that moreover there is no gap above the ground state.

We now briefly discuss five models or families of models, examples and counterexamples, and also indicate some open problems. It must be clear that our only aim is illustration and that what is given below definitely does not exhaust the possible applications of the theorems. We do not discuss any new examples of GVBS models with a unique ground state, because these are completely covered by the results in [14]. A recent addition to the family of GVBS models with unique ground states is e.g. [83].

We start with the well-known Majumdar-Ghosh model. Although it is a special case of the generalized Majumdar-Ghosh model discussed in example 1bis, we prefer to discuss it explicitly because it is the simplest GVBS model with more than one ground state.

Example 1. The Majumdar-Ghosh Model [49, 48]. Nothing new is to be proved about this model here, as it was already completely analyzed in [12]. But still it is a good starter because of its particular simplicity. The Majumdar-Ghosh model is a spin-1/2 chain with a nnn-interaction given by:

$$
H_{[0,2]}=h_{0}=P_{012}^{(3 / 2)}=\frac{2}{3}\left(\overrightarrow{\mathbf{S}}_{1} \cdot \overrightarrow{\mathbf{S}}_{2}+\overrightarrow{\mathbf{S}}_{2} \cdot \overrightarrow{\mathbf{S}}_{3}+\overrightarrow{\mathbf{S}}_{1} \cdot \overrightarrow{\mathbf{S}}_{3}\right)+\frac{1}{2} \mathbb{1}
$$

where $P_{012}^{(3 / 2)}$ is the orthogonal projection onto the subspace with total spin equal to $3 / 2$. At $T=0$ this model breaks the translation invariance of the chain and has two pure infinite-volume ground states which are fully dimerized: $\omega_{1}$ is a product of singlet states on $\mathrm{nn}$ pairs of the form $\{2 i, 2 i+1\}$ and $\omega_{2}$ is obtained from $\omega_{1}$ by translation over one lattice spacing. It is quite obvious that $\omega_{1}$ and $\omega_{2}$ are GVBS states. For a GVBS description of the unique translation invariant ground state $\frac{1}{2}\left(\omega_{1}+\omega_{2}\right)$ see [14], p. 472, Example 6 . In order to check the conditions of Theorem 2 it is convenient to consider the model on a regrouped chain where the new sites are now formed by $n n$ pairs of sites of the original chain. For concreteness put $\tilde{\mathscr{A}}_{l}=\mathscr{A}_{2 l} \otimes \mathscr{A}_{2 i+1}$ and we use to indicate any object related to the regrouped chain. Any interval of the regrouped chain corresponds to an interval of even length of the original chain, where it is easy to see that:

$$
\mathscr{G}_{[0,2 N+1]}^{(1)}=\mathbb{C}\left(\bigotimes_{i=0}^{N} \varphi_{2 l, 2 l+1}\right)
$$

where the superscript ${ }^{(1)}$ refers to $\omega_{1}$ and $\varphi_{2 i, 2 i+1}$ is the single state on the nn pair $\{2 i, 2 i+1\}$. For $\omega_{2}$ we have

$$
\mathscr{G}_{[0,2 N+1]}^{(2)}=\left\{\alpha \otimes \bigotimes_{l=0}^{N-1} \varphi_{2 i+1,2 i+2} \otimes \beta \mid \alpha, \beta \in \mathbb{C}^{2}\right\}
$$


So, the local support spaces $\tilde{\mathscr{G}}_{[M, N]}=\mathscr{G}_{[2 M, 2 N+1]}^{(1)} \vee \mathscr{G}_{[2 M, 2 N+1]}^{(2)}, N>M$, are 5-dimensional and it was shown explicitly in [12], that

$$
\operatorname{ker} H_{[2 M, 2 N+1]}=\tilde{\mathscr{G}}_{[M, N]} .
$$

Therefore all conditions of Theorem 2 are satisfied and the Majumdar-Ghosh model has a non-vanishing spectral gap in each of its ground states.

Example 1bis: Generalized Majumdar-Ghosh Models. The Majumdar-Ghosh model can be generalized to spins of arbitrary magnitude. The infinite volume ground states are then the two fully dimerized states of the spin- $S$ chain, which are just products of the singlet state of a nearest neighbour pair of spin $S$ 's. The Hamiltonian of a GVBS model with these ground states is clearly not unique. It is also not directly obvious that it will just contain nearest neighbour and next-nearest neighbour interactions and nothing else, but the computations of Long and Siak [6] show that interactions with further neighbours are, in fact, not needed. In terms of the total-spin projections $P_{l, j}^{(J)}$ of a pair of spins of magnitude $S$ at sites $i$ and $j$, the Hamiltonian studied in [6] reads:

$$
H=-\sum_{i}\left\{P_{l, i+1}^{(0)}+P_{l+1, i+2}^{(0)}+\frac{1}{2 S+1} \sum_{J=0}^{2 S}\left(1-(-1)^{2 S-J}\right) P_{i, i+2}^{(J)}\right\} .
$$

By adding the constant $(4 S+3) /(2 S+1)$ to the interaction term, its lowest eigenvalue can be made to vanish. Any other non-negative next-nearest-neighbour interaction with the same kernel defines a GVBS model with exactly the same ground states. The general results obtained in the present paper also apply to all these models and demonstrate the existence of a spectral gap above the ground state. The value of the spectral gap will, of course, depend on the specifics of the interaction chosen.

Example 2. A "critical" VBS-model. Our second example is a one-parameter family of Hamiltonians with nn interaction for a spin-1 chain showing spontaneous $\mathbb{Z}_{2}$-symmetry breaking. Let $\lambda \in[0,1]$ and define

$$
h_{i}^{(\lambda)}=\left(\mathbb{1}-\left(S_{i}^{z} S_{i+1}^{z}\right)^{2}\right)+\lambda\left(\mathbb{1}+\overrightarrow{\mathbf{S}}_{i} \cdot \overrightarrow{\mathbf{S}}_{i+1}-\left(S_{i}^{z}\right)^{2}-\left(S_{i+1}^{z}\right)^{2}+\left\{\overrightarrow{\mathbf{S}}_{i} \cdot \overrightarrow{\mathbf{S}}_{l+1}, S_{i}^{z} S_{i+1}^{z}\right\}\right),
$$

where $\{X, Y\} \equiv X Y+Y X$. In order to study the ground states of $H_{[M, N]}^{(\lambda)}=\sum_{i=M}^{N-1} h_{i}^{(\lambda)}$, we first have a look at the diagonalization of $h^{(\lambda)}$ acting on $\mathbb{C}^{3} \otimes \mathbb{C}^{3}$. As an orthonormal basis for $\mathbb{C}^{3}$ we take the eigenvectors of $S^{z}:|1\rangle,|0\rangle,|-1\rangle$. As a basis for $\mathbb{C}^{3} \otimes \mathbb{C}^{3}$ it is convenient to define

$$
\begin{aligned}
& \xi_{1}=|11\rangle, \quad \xi_{2}=|10\rangle+|01\rangle, \quad \xi_{3}=|10\rangle-|01\rangle, \\
& \xi_{4}=|1-1\rangle+|-11\rangle, \quad \xi_{5}=|00\rangle, \quad \xi_{6}=|1-1\rangle-|-11\rangle, \\
& \xi_{7}=|0-1\rangle-|-10\rangle, \quad \xi_{8}=|-10\rangle+|0-1\rangle, \quad \xi_{9}=|-1-1\rangle .
\end{aligned}
$$


It turns out that the $\xi_{j}$ are eigenvectors of $h^{(\lambda)}$ :

$$
\begin{aligned}
& h^{(\lambda)} \xi_{4}=0, \quad h^{(\lambda)} \xi_{6}=0, \\
& h^{(\lambda)} \xi_{3}=(1-\lambda) \xi_{3}, \quad h^{(\lambda)} \xi_{7}=(1-\lambda) \xi_{7}, \\
& h^{(\lambda)} \xi_{2}=(1+\lambda) \xi_{2}, \quad h^{(\lambda)} \xi_{5}=(1+\lambda) \xi_{5}, \quad h^{(\lambda)} \xi_{8}=(1+\lambda) \xi_{8}, \\
& h^{(\lambda)} \xi_{1}=2 \lambda \xi_{1}, \quad h^{(\lambda)} \xi_{9}=2 \lambda \xi_{9} .
\end{aligned}
$$

So, $\operatorname{spec} h^{(\lambda)}=\{0(2), 1-\lambda(2), 1+\lambda(3), 2 \lambda(2)\}$, where the numbers between parentheses denote the degeneracies, and $h^{(\lambda)} \geqq 0$ for all $\lambda \in[0,1]$. But $\operatorname{ker} h^{(\lambda)}$ depends upon whether $\lambda=1$ or $\lambda \in(0,1)$ or $\lambda=0$. It is now straightforward to determine $\operatorname{ker} H_{[M, N]}^{(\hat{i})}$ for all integers $M, N, N>M$. Define vectors $\Omega_{1}, \Omega_{2}, \Xi_{1}, \Xi_{2} \in\left(\mathbb{C}^{3}\right)^{\otimes N}$ by:

$$
\begin{aligned}
\Omega_{1}= & \left|1,-1, \ldots,(-1)^{N+1}\right\rangle, \quad \Omega_{2}=F^{\otimes N} \Omega_{1}, \\
\Xi_{1}= & \left|0,-1,1,-1, \ldots,(-1)^{N+1}\right\rangle-\left|-1,0,1,-1, \ldots,(-1)^{N+1}\right\rangle \\
& +\left|-1,1,0,-1, \ldots,(-1)^{N+1}\right\rangle-\cdots(-1)^{N+1}\left|-1,1, \ldots,(-1)^{N+1}, 0\right\rangle, \\
\Xi_{2}= & F^{\otimes N} \Xi_{1},
\end{aligned}
$$

where $F$ is the spin flip defined by $F|1\rangle=|-1\rangle, F|0\rangle=|0\rangle, F|-1\rangle=|1\rangle$. For $\lambda \in(0,1) \operatorname{ker} H_{[1, N]}^{(\lambda)}$ is 2-dimensional and spanned by $\Omega_{1}$ and $\Omega_{2}$. For $\lambda=1$ the kernel is 4 -dimensional and spanned by $\Omega_{1}, \Omega_{2}, \Xi_{1}$ and $\Xi_{2}$. For $\lambda=0$ the kernel is $2^{N}$-dimensional and spanned by all the Ising configurations (i.e. all configurations not containing any zeros). It is of course obvious that the spectrum of the model with $\lambda=0$ is entirely discrete and that there is a gap of magnitude 1 . But there is really nothing interesting in this model and we will not discuss it any further.

The infinite-volume ground states for any $\lambda \in(0,1]$ are just the ground states of the Ising antiferromagnet, obtained by extending $\Omega_{1}$ and $\Omega_{2}$ to the infinite chain. Call the respective thermodynamic limits $\omega_{1}$ and $\omega_{2}$. The thermodynamic limits of $\Xi_{1}$ and $\Xi_{2}$ are convex combinations of $\omega_{1}$ and $\omega_{2}$, depending on how exactly the finite interval is tending to $\mathbb{Z}$. So, we repeat, $\omega_{1}$ and $\omega_{2}$ (and the convex combinations of them) are the only infinite volume ground states for the model at $\lambda=1$. There is a difference however between the cases $\lambda<1$ and $\lambda=1$ : in the former case one has the property $\operatorname{ker} H_{[M, N]}^{(\lambda)}=\mathscr{G}_{[M, N]}$, where the $\mathscr{G}_{[M, N]}$ are the local support spaces for the state $\frac{1}{2}\left(\omega_{1}+\omega_{2}\right)$ (spanned by the two antiferromagnetic Ising configurations), whereas in the latter case one does not, because $\Xi_{1}$ and $\Xi_{2}$ are two additional finite volume ground states. So, if $\lambda<1$, Theorem 2 applies and there is a spectral gap above the ground state. We will now show that in the case $\lambda=1$ there is no gap and we will see that the low-lying excitations are closely related to the additional finite-volume ground states.

For $k \in \mathbb{R}$ and $N \geqq 1$ define the operators

$$
X_{N}^{\alpha}(k)=\sum_{-N \leqq x<y \leqq N} e^{i k(x-y)} S_{x}^{\alpha} e^{\left\{1 \pi \Sigma_{1<z<1} S_{z}^{\prime \prime}\right\}} S_{y}^{\alpha},
$$

where $\alpha \in\{x, y, z\}$ labels the three spin-1 operators. Note the similarity with the string order parameter employed by De Nijs and Rommelse in [84] and Kennedy and Tasaki in [76]. Denote by $\Omega_{i}^{M}, i=1,2$, the extensions of the vectors $\Omega_{i}$ to the 
interval $[-M, M]$. It is then easy to see that for $M \geqq N$ and $\alpha=x, y$

$$
\left\langle\Omega_{l}^{M} \mid X_{N}^{\alpha}(k) \Omega_{j}^{M}\right\rangle=0 .
$$

A straightforward computation yields

$$
\frac{\left\langle\Omega_{l}^{M} \mid X_{N}^{\alpha}(k)^{*} H_{[-M, M]}^{(1)} X_{N}^{\alpha}(k) \Omega_{i}^{M}\right\rangle}{\left\langle\Omega_{i}^{M} \mid X_{N}^{\alpha}(k)^{*} X_{N}^{\alpha}(k) \Omega_{i}^{M}\right\rangle}=\left(\frac{N-\frac{1}{3}}{N-\frac{1}{2}}\right) \frac{3}{4 N}+\left(\frac{2 N-1}{2 N+1}\right)(1-\cos k) .
$$

Hence the gap of $H_{[-M, M]}^{(1)}$ is $\mathbf{O}\left(\frac{1}{M}\right)$ and the infinite volume model has no gap.

Another gapless GVBS-model is the following. Let $\lambda \in[0,1]$ and define

$$
h_{i}^{(\lambda)}=\left(S_{l}^{z}\right)^{2}+\left(S_{l+1}^{z}\right)^{2}+\lambda\left\{-\mathbb{1}-\left(S_{l}^{z} S_{i+1}^{z}\right)^{2}+\frac{1}{2} \overrightarrow{\mathbf{S}}_{l} \cdot \overrightarrow{\mathbf{S}}_{i+1}+\frac{1}{2}\left(\overrightarrow{\mathbf{S}}_{i} \cdot \overrightarrow{\mathbf{S}}_{i+1}\right)^{2}\right\} .
$$

Define vectors $\Omega, \Xi_{1}, \Xi_{0}, \Xi_{-1} \in\left(\mathbb{C}^{3}\right)^{\otimes N}$ by:

$$
\begin{gathered}
\Omega=|0,0, \ldots, 0\rangle, \\
\Xi_{1}=|1,0,0, \ldots, 0\rangle-|0,1,0, \ldots, 0\rangle+|0,0,1,0, \ldots, 0\rangle+\cdots(-1)^{N+1}|0,0, \ldots, 0,1\rangle, \\
\Xi_{0}=\sum_{0<x<y<N}(-1)^{y-x}\left(S_{x}^{+} S_{y}^{-}-S_{x}^{-} S_{y}^{+}\right) \Omega, \\
\Xi_{-1}=F^{\otimes N} \Xi_{1},
\end{gathered}
$$

where $F$ is the spin flip defined by $F|1\rangle=|-1\rangle, F|0\rangle=|0\rangle, F|-1\rangle=|1\rangle$. For $\lambda \in[0,1) \operatorname{ker} H_{[1, N]}^{(\lambda)}=\mathbb{C} \Omega$ and for $\lambda=1$ the kernel becomes 4-dimensional and is spanned by $\Omega, \Xi_{1}, \Xi_{0}, \Xi_{-1}$. The only infinite volume ground state for any $\lambda \in[0,1]$ is the limit of the pure state determined by $\Omega$. For $\lambda<1$ there is a gap by the general theorem. For $\lambda=1$, there is no gap. It is easy to calculate e.g. the energy of a spin-wave polarized in the $\mathrm{x}$-direction. One finds the simple dispersion relation $E_{k}=1-\cos k$.

Example 3. Models with Helical Symmetry. Next we want to sketch briefly how simple models exhibiting helical symmetry breaking can be obtained. In one dimension, non-trivial helicity is as close as one can hope to get to the chiral symmetry breaking conjectured to occur in higher dimensions (see e.g. [5]).

We start from the spin-1 model introduced by Affleck, Kennedy, Lieb and Tasaki [11]. Its Hamiltonian is

$$
H^{\mathrm{AKLT}}=\sum_{i}\left\{\frac{1}{3}+\frac{1}{2} \overrightarrow{\mathbf{S}}_{i} \cdot \overrightarrow{\mathbf{S}}_{i+1}+\frac{1}{6}\left(\overrightarrow{\mathbf{S}}_{l} \cdot \overrightarrow{\mathbf{S}}_{l+1}\right)^{2}\right\} .
$$

The unique infinite volume ground state $\omega_{\mathrm{AKLT}}$ of the AKLT-model is the GVBSstate determined by

- the dimension of the auxiliary space is $k=2$ and the isometry $V$ is given by

$$
\begin{gathered}
V\left|\frac{1}{2}\right\rangle=\sqrt{\frac{2}{3}}\left|1,-\frac{1}{2}\right\rangle-\sqrt{\frac{1}{2}}\left|0, \frac{1}{2}\right\rangle, \\
V\left|-\frac{1}{2}\right\rangle=\sqrt{\frac{1}{3}}\left|0,-\frac{1}{2}\right\rangle-\sqrt{\frac{2}{3}}\left|-1, \frac{1}{2}\right\rangle .
\end{gathered}
$$




$$
\begin{aligned}
& -\mathbb{E}_{A}(B)=V^{*} A \otimes B V \text { for all } A \in \mathscr{M}_{3}, B \in \mathscr{M}_{2} . \\
& -\omega_{\mathrm{AKLT}}\left(A_{1} \otimes \cdots \otimes A_{n}\right)=\frac{1}{2} \operatorname{Tr} \mathbb{E}_{A_{1}} \circ \cdots \circ \mathbb{E}_{A_{n}}(\mathbb{1}) .
\end{aligned}
$$

Our aim is to perturb $\omega_{\mathrm{AKLT}}$ in such a way that part of the rotation invariance is broken and a state with non-trivial helicity is obtained. It is then straightforward to construct a Hamiltonian which has two ground states with opposite helicity. Such a model can also be considered as an example of a model where the reflection symmetry of the chain is spontaneously broken.

We will denote by $S^{x}, S^{y}, S^{z}$ the usual spin-1 matrices and by $J^{x}, J^{y}, J^{z}$ the spin$\frac{1}{2}$ matrices, which generate the 3-dimensional irreducible representation $D^{(1)}$ and the 2-dimensional irreducible representation $D^{(1 / 2)}$ of SU(2) respectively. The isometry $V$ intertwines the representations $D^{(1)} \otimes D^{(1 / 2)}$ and $D^{(1 / 2)}$, and hence

$$
\mathbb{E}_{A}\left(D^{\left(\frac{1}{2}\right)}(g) B D^{\left(\frac{1}{2}\right)}(g)^{*}\right)=D^{\left(\frac{1}{2}\right)}(g) \mathbb{E}_{D^{(1)}(g)^{*} A D^{(1)}(g)}(B) D^{\left(\frac{1}{2}\right)}(g)^{*} .
$$

For $\alpha \in[0,4 \pi)$ define:

$$
U(\alpha)=e^{i \alpha J^{z}}=\left(\begin{array}{cc}
e^{i \alpha / 2} & 0 \\
0 & e^{-i \alpha / 2}
\end{array}\right)
$$

and put

$$
\mathbb{E}_{A}^{(\alpha)}(B)=\mathbb{E}_{A}\left(U(\alpha) B U(\alpha)^{*}\right) .
$$

A family of new GVBS-states is defined by

$$
\omega_{\alpha}\left(A_{1} \otimes \cdots \otimes A_{n}\right)=\frac{1}{2} \operatorname{Tr} \mathbb{E}_{A_{1}}^{(\alpha)} \circ \cdots \circ \mathbb{E}_{A_{n}}^{(\alpha)}(\mathbb{1}) .
$$

Using (6.1) it is straightforward to check the following relation between $\omega_{\text {AKLT }}$ and $\omega_{\alpha}$ :

$$
\begin{aligned}
& \omega_{\alpha}\left(A_{1} \otimes \cdots \otimes A_{n}\right) \\
& =\omega_{\mathrm{AKLT}}\left(R((n-1) \alpha)^{*} A_{1} R((n-1) \alpha) \otimes R((n-2) \alpha)^{*} A_{1} R((n-2) \alpha) \otimes \cdots \otimes A_{n}\right),
\end{aligned}
$$

where $R(\beta)=\exp i \beta S^{z}$ for all $\beta \in \mathbb{R}$. So, $\omega_{x}$ is obtained from $\omega_{\mathrm{AKLT}}$ by a "twist" about the $z$-axis over an angle $\alpha$ per lattice spacing. Obviously $\omega_{0}=\omega_{2 \pi}=\omega_{\text {AKLT }}$. Equation (6.1) expresses the rotation invariance of $\omega_{\mathrm{AKLT}}$ and implies that the state $\omega_{\alpha}$ is still translation invariant. However the rotation invariance is reduced to rotations about the $z$-axis only. The $\omega_{x}$ are the unique ground states of a family of Hamiltonians obtained from $H^{\text {AKLT }}$ by the corresponding "twist." More interesting is the fact that from Theorem 1 it follows that, if $\alpha \neq-\alpha \bmod 2 \pi$, we can also find a finite range interaction, say $h^{(\alpha)}$, such that the corresponding model has exactly two ground states: $\omega_{\alpha}$ and $\omega_{-\alpha}$. It is in principle absolutely straightforward to obtain explicit expressions for the interactions $h^{(\alpha)}$ by computing the local support spaces of the states $\omega_{\alpha}$ and by determining a value of $m_{0}$ for which the intersection property (3.10) holds. The actual computation might be somewhat tedious and not particularly enlightening. The result is a model of the form

$$
H^{(\alpha)}=\sum_{i}\left(\mathbb{1}-G_{\left[1+i, m_{0}+i\right]}^{(\alpha)}\right) .
$$

Theorem 2 implies the existence of a spectral gap above the ground state. 
Let us end the discussion of the states $\omega_{\alpha}$ by computing the various order parameters and correlation functions that are usually employed to reveal the structure of quantum spin states, in particular the ones that were investigated in the recent literature on spin-1 chains $[84,12,76]$. We define as usual $J^{ \pm}=J^{x} \pm i J^{y}$. All expectation values in $\omega_{\alpha}$, can be calculated using (3.4) and Table 1, which fully describes the operator $\mathbb{E}^{(\alpha)}$.

1) The magnetization vanishes:

$$
\omega_{\alpha}\left(S_{0}^{\gamma}\right)=0 \text { for } \gamma=x, y, z .
$$

2) The spin-spin correlation functions are periodic but not necessarily commensurate with the lattice:

$$
\begin{aligned}
& \omega_{\alpha}\left(S_{0}^{z} S_{r}^{\gamma}\right)=\omega_{\alpha}\left(S_{0}^{\gamma} S_{r}^{z}\right)=\delta_{\gamma, z} \frac{4}{3}\left(-\frac{1}{3}\right)^{r}, \quad r \geqq 1, \\
& \omega_{\alpha}\left(S_{0}^{x} S_{r}^{x}\right)=\omega_{\alpha}\left(S_{0}^{y} S_{r}^{y}\right)=\frac{4}{3}\left(-\frac{1}{3}\right)^{r} \cos r \alpha, \quad r \geqq 1, \\
& \omega_{\alpha}\left(S_{0}^{x} S_{r}^{y}\right)=-\omega_{\alpha}\left(S_{0}^{y} S_{r}^{x}\right)=\frac{4}{3}\left(-\frac{1}{3}\right)^{r} \sin r \alpha, \quad r \geqq 1 .
\end{aligned}
$$

3) The den Nijs-Rommelse string order parameter according to its original definition in [84] is given by:

$$
O^{\gamma}=\lim _{r \rightarrow \infty} \omega_{\alpha}\left(S_{0}^{\gamma} \prod_{x=1}^{r-1} e^{i \pi S_{i}^{i}} S_{r}^{\gamma}\right),
$$

and we have

$$
\begin{array}{ll}
O^{z}=O^{x}=O^{y}=\frac{1}{9} & \text { if } \alpha=k \pi, k \in \mathbb{Z}, \\
O^{z}=\frac{1}{9}, \quad O^{x}=O^{y}=0 & \text { else. }
\end{array}
$$

4) The helicity of the state can be measured by the correlation function $\chi^{z}(r)=$ $S_{0}^{x} S_{r}^{y}-S_{0}^{y} S_{r}^{x}$. The states $\omega_{\alpha}$ have short range helicity but no long range helical order. From 2) it follows that $\chi(1)=-\frac{4}{9} \sin r \alpha \neq 0$ if $\alpha \neq k \pi$, but $\lim _{r \rightarrow \infty} \chi(r)=0$.

Example 4. A Model with Charge Conjugation Symmetry Breaking. Finally we consider a model with spontaneous breaking of the charge conjugation symmetry that was first presented by Affleck, Arovas, Marston and Rabson in [7].We will call it the AAMR-model. As the construction of the model and the analysis of its properties is based on the structure of the irreducible representations of SU(4), we have to recall some of the basic facts about these first. For more information see e.g. [85].

The irreducible representations of SU(4) are labeled by the Young tableaux with three rows, including the empty tableau (or alternatively the Young tableau consisting of a single column of four boxes) which stands for the trivial representation or, in physical terms, the singlet. The number of boxes in each row are denoted by integers $v_{1}, v_{2}, v_{3}$, satisfying $v_{1} \geqq v_{2} \geqq v_{3} \geqq 0$, and $\left[v_{1}, v_{2}, v_{3}\right]$ is an alternative way to denote a particular irreducible representation. Using Robinson's formula [85] one easily obtains the following expression for the dimension of an irreducible representation:

$$
\operatorname{dim}\left[v_{1}, v_{2}, v_{3}\right]=\frac{1}{12}\left(v_{1}+3\right)\left(v_{2}+2\right)\left(v_{3}+1\right)\left(v_{1}-v_{2}+1\right)\left(v_{1}-v_{3}+2\right)\left(v_{2}-v_{3}+1\right),
$$


Table 1. Values taken by the bilinear operator $\mathbb{E}_{A}^{(\alpha)}(B)$ on the basis of spin matrices.

\begin{tabular}{lllll}
\hline $\mathbb{E}_{A}^{(\alpha)}(B)$ & $B=\mathbb{1}$ & $B=J^{z}$ & $B=J^{+}$ & $B=J^{-}$ \\
\hline$A=\mathbb{1}$ & $\mathbb{1}$ & $-\frac{1}{3} J^{z}$ & $-\frac{1}{3} e^{i \alpha} J^{+}$ & $-\frac{1}{3} e^{-l \alpha} J^{-}$ \\
$A=S^{z}$ & $\frac{4}{3} J^{z}$ & $-\frac{1}{3} \mathbb{1}$ & 0 & 0 \\
$A=S^{+}$ & $\frac{4}{3} J^{+}$ & 0 & $-\frac{2}{3} e^{i \alpha} \mathbb{1}$ & 0 \\
$A=S^{-}$ & $\frac{4}{3} J^{-}$ & 0 & 0 & $-\frac{2}{3} e^{-i \alpha} \mathbb{1}$ \\
\hline
\end{tabular}

e.g. in what follows we will use

$$
\operatorname{dim} \square=6, \quad \operatorname{dim} \square \square=20 .
$$

The decomposition of a tensor product of two irreducible representations into a direct sum of irreducible representations is given by the usual rule for multiplying Young tableaux with four rows and using the equivalence $\left[v_{1}, v_{2}, v_{3}, v_{4}\right] \equiv\left[v_{1}-v_{4}\right.$, $\left.v_{2}-v_{4}, v_{3}-v_{4}\right]$. We will e.g. need:

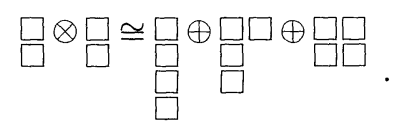

$\mathrm{SU}(4)$ is a 15-dimensional Lie group, but it is convenient to represent its Lie algebra as the traceless subalgebra of the Lie algebra of U(4), i.e. we consider generators $S_{\beta}^{\alpha}, 1 \leqq \alpha, \beta \leqq 4$ satisfying

$$
\left[S_{\beta}^{\alpha}, S_{v}^{\mu}\right]=\delta_{\beta}^{\mu} S_{v}^{\alpha}-\delta_{v}^{\alpha} S_{\beta}^{\mu}
$$

with the constraint that $\operatorname{Tr} S=\sum_{\alpha} S_{\alpha}^{\alpha}=0$ and the $S_{\beta}^{\alpha}$ are chosen such that $\left(S_{\beta}^{\alpha}\right)^{*}=$ $S_{\alpha}^{\beta}$. From any representation of this Lie algebra, say generated by $S_{\beta}^{\alpha}$, we can obtain another one generated by $S_{\beta}^{\prime \alpha}$ by putting $S_{\beta}^{\prime \alpha}=-S_{\alpha}^{\beta}$. This is the conjugate representation for which we will systematically use primed quantities. Of course there is a corresponding conjugation operation for the irreducible representations of $\mathrm{SU}(4)$ and hence for the Young tableaux. It is described by $\left[v_{1}, v_{2}, v_{3}\right]^{\prime}=\left[v_{1}, v_{1}-v_{3}, v_{1}-v_{2}\right]$, e.g. :

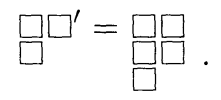

In general mutually conjugate representations are not equivalent (see e.g. the above example), but some are:

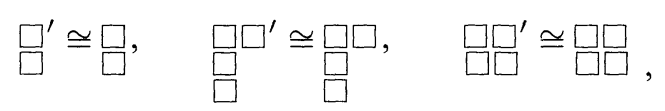


and of course

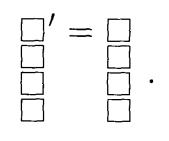

A self-conjugate representation and its conjugate are isomorphic, but not identical (except of course for the case of the singlet representation) and hence there is a non-trivial unitary $C$ implementing this isomorphism which is called the charge conjugation operator. $C$ is a spontaneously broken symmetry in the AAMR-model, which we will introduce now.

The one-site Hilbert space of the AAMR-model is $\mathbb{C}^{6}$ on which one lets $\mathrm{SU}(4)$ act by its 6 -dimensional irreducible representation $[1,1,0]$. So, for each pair of sites the irreducible representations that appear are given by the decomposition (6.2). Let $P^{(\boxplus)}$ denote the orthogonal projection onto the subspace of $\mathbb{C}^{6} \otimes \mathbb{C}^{6}$ supporting the irreducible representation $[2,2,0]$. The Hamiltonian of the AAMR-model is then:

$$
H_{[M, N]}=\sum_{l=M}^{N-1} P^{(\text {(日) })} .
$$

As all representations in the decomposition (6.2) are self-conjugate and distinct, it is obvious that the projection operators onto their supports commute with $C$, and hence the Hamiltonian (6.3) is charge conjugation symmetric. We now construct two distinct pure ground states for the model, which are both SU(4)-invariant and related to each other by charge conjugation. This implies that the model exhibits spontaneous breaking of the charge conjugation symmetry. These two ground states are given in [7] in a convenient representation using fermion operators in four flavors. As our main purpose here is to see how the general results of this paper apply to this model, we prefer to give a more compact definition of these states as GVBS-states.

Consider two isometries $V$ and $V^{\prime}: \mathbb{C}^{4} \rightarrow \mathbb{C}^{6} \otimes \mathbb{C}^{4}$ satisfying the intertwining relations

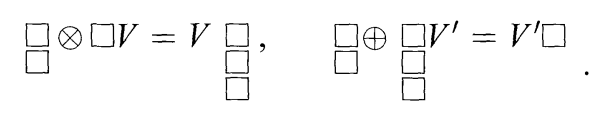

The decomposition

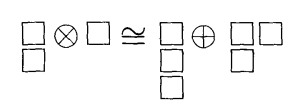

and the conjugate of this relation, imply that these isometries exist and are unique up to a phase. For all $A \in \mathscr{M}_{6}$ we then define the transformations $\mathbb{E}_{A}$ and $\mathbb{E}_{A}^{\prime}$ of $\mathscr{M}_{4}$ by

$$
\begin{aligned}
& \mathbb{E}_{A}(B)=V^{*} B V, \\
& \mathbb{E}_{A}^{\prime}(B)=V^{*} B V^{\prime} .
\end{aligned}
$$

Because in this model not only the charge conjugation symmetry but also translation invariance is spontaneously broken, it is convenient to consider a regrouped chain, where the new sites consist of pairs of nearest neighbour sites of the original chain. 
Quantities referring to the regrouped chain will be denoted by . Obviously any state $\omega$ of the regrouped chain is a state of the original chain and vice versa. Two GVBS-states $\omega$ and $\omega^{\prime}$ are defined by:

$$
\begin{aligned}
\omega\left(A_{1} \otimes \cdots \otimes A_{2 n}\right) & =\frac{1}{4} \operatorname{Tr} \mathbb{E}_{A_{1}} \circ \mathbb{E}_{A_{2}}^{\prime} \circ \cdots \circ \mathbb{E}_{A_{2 n-1}} \circ \mathbb{E}_{A_{2 n}}^{\prime}(\mathbb{1}), \\
\omega\left({ }^{\prime} A_{1} \otimes \cdots \otimes A_{2 n}\right) & =\frac{1}{4} \operatorname{Tr} \mathbb{E}_{A_{1}}^{\prime} \circ \mathbb{E}_{A_{2}} \circ \cdots \circ \mathbb{E}_{A_{2 n-1}}^{\prime} \circ \mathbb{E}_{A_{2 n}}^{\prime}(\mathbb{1}) .
\end{aligned}
$$

It is quite obvious that the trace is invariant under $\mathbb{E}_{\mathbb{1}}$ and $\mathbb{E}_{\mathbb{1}}^{\prime}$, and so $\omega \circ \tau=\omega^{\prime}$, where $\tau$ is the translation over one lattice spacing. It is also evident that $\omega$ and $\omega^{\prime}$ are related to one another by charge conjugation, as $V$ and $V^{\prime}$ are. The fact that charge conjugation symmetry is broken can also be expressed by a non-vanishing order parameter (see [7]). That $\omega$ and $\omega{ }^{\prime}$ are ground states of the model follows from the transformation properties of $\omega \mathscr{A}_{[1,2]}$ and $\omega^{\prime} \mid \mathscr{A}_{[1,2]}$. One readily sees that the support of the density matrices describing the restriction of the state to a pair of nearest neighbour points transform as

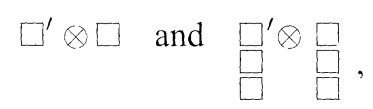

which decompose as

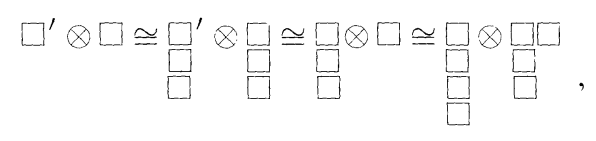

and so the supports do not contain $[2,2,0]$, and hence $\omega(H)=\omega^{\prime}(H)=0$ and $\omega$ and $\omega^{\prime}$ are ground states.

We now would like to apply Theorem 2 to get the existence of a spectral gap in the AAMR-model. The condition one has to check is the following: we have to verify that for some large enough interval $[1, l]$, all zero energy vectors of $H_{[1, l]}$ are in the supports of $\omega$ and $\omega^{\prime}$. This is claimed in [7] but we do not have a complete argument for this property. It would nice to have effective techniques to check these kind of properties for this and more general models.

Acknowledgements. The author would like to thank Ian Affleck, Michael Aizenman, Joe Conlon, Mark Fannes, Ellott Lieb, Fabio Martinelli, Hal Tasaki, Reinhard Werner, Horng-Tzer Yau, and Boguslaw Zegarlinski for interesting discussions and/or useful comments on the literature. Theorem 3 is based on a discussion with Horng-Tzer Yau. This work has benefited from a travel grant of the Nationaal Fonds voor Wetenschappelijk Onderzoek, Belgium, and is financially supported in part by NSF Grants \# PHY-8912067 and \# PHY90-19433 A03.

\section{References}

1. Affleck, I.: Large- $n$ limit of SU( $n)$ quantum "spin" chains. Phys. Rev. Lett 54, 966-969 (1985)

2. Affleck, I.: Exact results on the dimerization transition in $\mathrm{SU}(n)$ antiferromagnetic chains. J. Phys.:Condens. Matter 2, 405-415 (1990)

3. Kennedy, T., Tasaki, H.: Hidden $Z_{2} \times Z_{2}$ symmetry breaking in Haldane gap antiferromagnets. Phys. Rev B45, 304-307 (1992)

4. Dagotto, E.: The $\mathrm{t}-\mathrm{J}$ and frustrated Heisenberg model: a status report on numerical studies. Int. I. Mod. Phys B5 907-935 (1991) 
5. Wen, X.G., Wilczeck, F., Zee, A.: Chiral spin states and superconductivity. Phys. Rev B39, 11413-11423 (1989)

6. Long, M.W., Siak, S.: An exact solution to a spin-1 chain model. J. Phys.: Condens. Matter 5, 5811-5828 (1993)

7. Affleck, I., Arovas, D.P., Marston, J.B., Rabson, D.A.: SU(2n) Quantum Antiferromagnets with Exact C-Breaking Ground States. Nucl. Phys B366, 467-506 (1991)

8. Affleck, I., Haldane, F.D.M.: Critical theory of quantum spin chains. Phys. Rev. B36, 52915300 (1987)

9. Affleck, I.: Quantum spin chains and the Haldane gap. J. Phys.:Condens. Matter 1, 3047-3072 (1989)

10. Sørensen, E.S., Affleck, I.: Large-Scale Numerical Evidence for Bose Condensation in the $S=1$ Antiferromagnetic Chain in a Strong Field. Phys. Rev. Lett. 71, 1633-1636 (1993)

11. Affleck, I., Lieb, E.H., Kennedy, T., Tasaki H.: Rigorous results on valence-bond ground states in antiferromagnets. Phys. Rev. Lett. 59, 799-802 (1987)

12. Affleck, I., Kennedy, T., Lieb, E.H., Tasaki H.: Valence bond ground states in isotropic quantum antiferromagnets. Commun. Math. Phys. 115, 477-528 (1988)

13. Fannes, M., Nachtergaele, B., Werner, R.F.: Valence bond states on quantum spin chains as ground states with spectral gap. J. Phys. A: Math. Gen. 24 L185-L190 (1991)

14. Fannes, M., Nachtergaele, B., Werner, R.F.: Finitely Correlated States on Quantum Spin Chains. Commun. Math. Phys. 144, 443-490 (1992)

15. Arovas, D.P., Auerbach, A., Haldane, F.D.M.: Extended Heisenberg models of antiferromagnetism: analogies to the fractional quantum Hall effect. Phys. Rev. Lett. 60, 531-534 (1988)

16. Knabe, S.: Energy gaps and elementary excitations for Certain VBS-Quantum Antiferromagnets. J. Stat. Phys. 52, 627-638 (1988)

17. Botet, R., Julien. R.: Ground-state properties of a spin-1 antiferromagnetic chain. Phys. Rev. B27, 613-615 (1983)

18. Kolb, M., Botet, R., Julien, J.: Comparison of ground-state properties for odd half-integer and integer spin antiferromagnetic Heisenberg chains. J. Phys. A: Math. Gen. 16, L673-L677 (1983)

19. Parkinson, J.B., Bonner, J.C.: Spin chains in a field: Crossover form quantum to classical behavior. Phys. Rev. B32, 4703-4724 (1985)

20. Nightingale, M.P., Blöte, H.W.: Gap of the linear spin-1 Heisenberg antiferromagnet: a Monte Carlo calculation. Phys. Rev. B33, 659-661 (1986)

21. Sólyom, J.: Competing bilinear and biquadratic exchange couplings in spin-1 Heisenberg chains. Phys. Rev. B36, 8642--8648 (1987)

22. Chang, K., Affleck, I., Hayden, G.W., Soos, Z.G.: A study of the bilinear-biquadratic spin 1 antiferromagnetic chain using the valence-bond basis. J. Phys. C1, 153-167 (1989)

23. Kennedy, T.: Exact diagonalisations of open spin-1 chains. J. Phys.: Cond. Matter 2, 5737-5745 (1990)

24. White, S.R., Huse, D.A.: Numerical Renormalization Group Study of Low-lying Eigenstates of the Antiferromagnetic $S=1$ Heisenberg Chain. Phys. Rev. B48, 3844-3852 (1993)

25. White, S.R.: Density Matrix Formulation for Quantum Renormalization Groups. Phys. Rev. Lett. 69, 2863-2866 (1992)

26. White, S.R.: Density Matrix Formulation for Quantum Renormalization Groups. Phys. Rev. B48, 10345-10456 (1993)

27. Kennedy, T.: Nonpositive matrix elements for Hamiltonians of spin 1 chains. J. Phys.: Cond. Matter 6, 8015-8022 (1994)

28. Caspers, W.J., Magnus, W.: Some exact excited states in a linear antiferromagnetic spin system. Phys. Lett. 88A 103-105 (1982)

29. Shastry, B.S., Sutherland, B.: Excitation Spectrum of a Dimerized Next-Neighbor Antiferromagnetic Chain. Phys. Rev. Lett. 47, 964-967 (1981)

30. Holley, R.: Rapid convergence to equilibrium in one-dimensional stochastic Ising models. Ann. Prob. 13, 72-89 (1985)

31. Aizenman, M., Holley, R.: Rapid convergence to equilibrium of stochastic Ising models in the Dobrushin-Shlosman regime. In: Percolation Theory and Ergodic Theory of Infinite Particle Systems, H. Kesten (Ed.), Berlin-Heidelberg-New York: Springer Verlag, 1987, pp. 1-11

32. Lu, S.-L., Yau, H.T.: Spectral gap and logarithmic Sobolev Inequality for Kawasaki and Glauber dynamics. Commun. Math. Phys. 156, 399-433 (1993) 
33. Martinelli, F., Olivieri, E.: Finite volume mixing conditions for lattice spin systems and exponential approach to equilibrium of Glauber dynamics. Parts I and II, Commun. Math. Phys. 161, 447-486, 487-514 (1994)

34. Holley, R.A., Stroock, D.W.: Logarithmic Sobolev Inequalities and stochastic Ising models. J. Stat. Phys. 46, 1159-1194 (1987)

35. Holley, R.A., Stroock, D.A.: Uniform and $L^{2}$ convergence in one-dimensional stochastic Ising models. Commun. Math. Phys. 123, 85-93 (1989)

36. Zegarlinski, B.: Gibbsian description and description by stochastic dynamics in the statistical mechanics of lattice spin systems with finite range interactions. In: Proceedings of the Third International Conference on Stochastic Processes, Physics, and Geometry, S. Albeverio et al. (Eds), Singapore: World Scientific, 1993

37. Holley, R.: Rapid convergence to equilibrium in ferromagnetic stochastic Ising models. Resenhas IME-USP 1, 131-149 (1993)

38. Zegarlinski, B.: Log-Sobolev inequlaities for infinite one-dimensional lattice systems. Commun. Math. Phys. 133, 147-162 (1990)

39. Stroock, D.W., Zegarlinski, B.: The logarithmic Sobolev inequality for continuous spin systems on a lattice. J. Funct. Anal. 104, 299-326 (1992)

40. Stroock, D.W., Zegarlinski, B.: The equivalence of the logarithmic Sobolev inequality and the Dobrushin-Shlosman mixing condition. Commun. Math. Phys. 144, 303-323 (1992)

41. Stroock, D.W., Zegarlinski, B.: The logarithmic Sobolev inequality for discrete spin systems on a lattice. Commun. Math. Phys. 149, 175-193 (1992)

42. Haldane, F.D.M.: The Hierarchy of Fractional States and Numerical Studies. In R.E. Prange, S.M. Girvin (eds.): The Quantum Hall Effect New York: Springer Verlag, 1987, pp. 303-352

43. Fröhlich, J., Studer, U.M.: U(1) $\times \mathrm{SU}(2)$ gauge invariance of non-relativistic quantum mechanics, and generalized Hall effects. Commun. Math. Phys. 148, 553-600 (1992)

44. Fannes, M., Nachtergaele, B., Werner, B.F.: Exact Ground States of Quantum Spin Chains Europhys. Lett. 10, 633-637 (1989)

45. Werner, R.F.: Remarks on a quantum state extension problem. Lett. Math. Phys. 19, 319-326 (1990)

46. Haldane, F.D.M.: Continuum dynamics of the 1-D Heisenberg antiferromagnet: Identification with the $\mathrm{O}(3)$ nonlinear sigma model. Phys. Lett. 93A, 464-468 (1983)

47. Affleck, I., Lieb, E.H.: A proof of part of Haldane's conjecture on quantum spin chains. Lett. Math. Phys. 12, 57-69 (1986)

48. Majumdar, C.K.: Antiferromagnetic model with known ground state. J. Phys. C: Cond Matt. 3, 911-915 (1970)

49. Majumdar, C.K., Ghosh, D.K.: On next nearest-neighbor interaction in linear chain, I and II. J. Math. Phys. 10, 1388-1398, and 1399-1402 (1969)

50. Klein, D.J.: Variational localized-site cluster expansions. IX. Many-body valence-bond theory. Phys. Rev. B19, 870-876 (1979)

51. van den Broeck, P.M.: Exact value of the ground state energy of the linear antiferromagnetic Heisenberg chain with nearest and next-nearest neighbor interactions. Phys. Lett. 77A, 261-262 (1980)

52. Klein, D.J.: Exact ground states for a class of antiferromagnetic Heisenberg models with short range interactions. J. Phys. A: Math. Gen. 15, 661-671 (1982)

53. Caspers, W.J.: Exact ground states for a class of linear antiferromagnetic spin systems. Physica 115A, 275-280 (1982)

54. Caspers, W.J., Magnus, W.: Exact ground states for a class of linear quantum spin systems. Physica 119A, 291-294 (1983)

55. Fannes, M., Nachtergaele, B., Werner, B.F.: Entropy Estimates for Finitely Correlated States. Ann. Inst. H. Poincaré 57, 259-277 (1992)

56. Bose, I.: Exact ground and excited states of an antiferromagnetic quantum spin model. J. Phys.: Condens. Matt. 1, 9267-9271 (1989)

57. Klümper, A., Schadschneider, A., Zittartz, J.: Ground state properties of a generalized VBSmodel. Z. Phys. B-Condensed Matter 87, 281-287 (1992)

58. Kennedy, T., Lieb, E.H., Tasaki, H.: A two-dimensional isotropic quantum antiferromagnet with unique disordered ground state. J. Stat. Phys. 53, 383-415 (1988)

59. Chayes, J., Chayes, L., Kivelson, S.: Valence bond ground states in a frustrated twodimensional spin 1/2 Heisenberg antiferromagnet. Commun. Math. Phys. 123, 53-83 (1989) 
60. Kirillov, A.N., Korepin, V.E.: The resonating valence bond in quasicrystals. Leningrad Math. J. 1, 343- 377 (1990)

61. Long, M.W., Siak, S.: An exactly soluble two-dimensional quantum mechanical Heisenberg model: quantum fluctuations versus magnetic order. J. Phys.: Condens. Matter 2, 10321-10341 (1990)

62. Bose, I.: Two-dimensional spin models with resonating valence bond ground states. J. Phys.: Consdens. Matter 2, 5479-5482 (1990)

63. Bose, I.: Frustrated spin $\frac{1}{2}$-model in two dimensions with a known ground state. Phys. Rev. B44, 443-445 (1991)

64. Bose, I.: Antiferromagnetic spin models in two dimensions with known ground states. Phys. Rev. B45, 13072-13075 (1992)

65. Freitag, W.-D., Müller-Hartmann, E.: Complete analysis of two-spin correlations of valence bond solid chains for all integer spins. Z. Phys. B Condensed Matter 83, 381-390 (1991)

66. Freitag, W.-D., Müller-Hartmann, E.: Spin correlations of inhomogeneous valence bond solid chains. Z. Phys. B Condensed Matter 88, 279-282 (1992)

67. Accardi, L.: Topics in Quantum Probability. Physics Rep. 77, 169-192 (1981)

68. Accardi, L., Frigerio, A.: Markovian Cocycles. Proc. R. Ir. Acad. 83A (2), 251-263 (1983)

69. Fannes, M., Nachtergaele, B., Werner, R.F.: Abundance of Translation Invariant Pure States on Quantum Spin Chains. Lett. Math. Phys. 25, 249-258 (1992)

70. Fannes, M., Nachtergaele, B., Werner, R.F.: Finitely correlated pure states. J. Funct. Anal. 120, 511-534 (1994)

71. Monti, F., Sütő, A.: Spin 1/2 Heisenberg model on $\Delta$ trees. Phys. Lett. 156, 197-200 (1991)

72. Monti, F., Sütő, A.: Heisenberg Antiferromagnet on Triangulated Trees. Helv. Phys. Acta 65, 560-595 (1992)

73. Gottstein, C.-T., Werner, R.F.: Ground states of the infinite q-deformed Heisenberg ferromagnet. Preprint archived as cond-mat/9501123

74. Albanese, C.: Unitary Dressing Transformations and Exponential Decay Below Threshold for Quantum Spin Systems. Part I-II. Commun. Math. Phys. 134, 1-27 (1990)

75. Albanese, C.: Unitary Dressing Transformations and Exponential Decay Below Threshold for Quantum Spin Systems. Part III-IV. Commun. Math. Phys. 134, 237-272 (1990)

76. Kennedy, T., Tasaki, H.: Hidden Symmetry Breaking and the Haldane Phase in $\mathrm{S}=1$ Quantum Spin Chains. Commun. Math. Phys. 147, 431-484 (1992)

77. Matsui, T.: Purification and Uniqueness of Quantum Gibbs States. Commun. Math. Phys. 162, 321-332 (1994)

78. Datta, N., Fernández, R., Fröhlich, J.: Low temperature phase diagrams of quantum lattice systems I. Texas archive 95-288

79. Koma, T., Tasaki, H.: Symmetry Breaking and Finite-Size Effects in Quantum Many-Body Systems. J. Stat. Phys. 76, 745-803 (1994)

80. Werner, R.F.: Finitely correlated pure states. In: M. Fannes, C. Maes, and A. Verbeure (eds), On three levels; micro-, meso, and macro-approaches in physics. New York: Plenum, 1994

81. Hiai, F., Petz, D.: Entropy Density for Algebraic States. J. Funct. Analysis 125, 287-308 (1994)

82. Olivieri, E., Picco, P.: Cluster Expansion for $d$-Dimensional Lattice Systems and Finite-Volume Factorization Properties. J. Stat. Phys. 59, 221-256 (1990)

83. Lange, C., Klümper, A., Zittartz, J.: Exact ground states for antiferromagnetic spin-one chains with nearest and next-nearest neighbour interactions. Preprint

84. den Nijs, M., Rommelse, K.: Preroughening transitions in crystal surfaces and valence-bond phases in quantum spin chains. Phys. Rev B40, 4709 (1989)

85. Chen, J.-Q.: Group Representation Theory for Physicists, Singapore: World Scientific, 1989

Communicated by D. Brydges

Note added in proof. Almost simultaneously with Ref. 78 another preprint containing essentially the same result appeared: Borgs, C., Kotecky, R., Ueltschi, D.: Low temperature phase diagrams for quantum perturbations of classical spin systems. Texas archive 95-233 